\title{
Dynamic Analysis and Synchronization with Unidirectional Coupling Involving Energy of a HR Neuron Under Electric Field and Memristive Autapse
}

\author{
Shuai Qiao \\ Northwest Normal University \\ Chenghua Gao ( $\nabla$ gaokuguo@163.com ) \\ Northwest Normal University https://orcid.org/0000-0001-8677-3853 \\ Xinlei An \\ Lanzhou Jiaotong University \\ Xingyue $\mathrm{He}$ \\ Northwest Normal University \\ Jingjing Wang \\ Northwest Normal University
}

\section{Research Article}

Keywords: Memristive autapse, Bistable, Multistability, Hamilton energy, Synchronization

Posted Date: October 29th, 2021

DOI: https://doi.org/10.21203/rs.3.rs-994955/v1

License: (c) (i) This work is licensed under a Creative Commons Attribution 4.0 International License.

Read Full License 


\title{
Dynamic Analysis and Synchronization with Unidirectional Coupling Involving Energy of A HR Neuron Under Electric Field and Memristive Autapse
}

\author{
Shuai Qiao ${ }^{1}$, Chenghua $\mathrm{Gao}^{1 *}$, Xinlei $\mathrm{An}^{2,3}$, Xingyue $\mathrm{He}^{1}$, Jingjing Wang ${ }^{1}$ \\ 1. College of Mathematics and Statistics, Northwest Normal University, Lanzhou 730070, China; \\ 2. School of Mathematics and Physics, Lanzhou Jiaotong University, Lanzhou,730070, China; \\ 3. College of Electrical and Information Engineering, Lanzhou University of Technology, Lanzhou \\ 730050, China.
}

Abstract: Reliable neuron models play an important role in identifying the electrical activities, global bifurcation patterns, and dynamic mechanisms of neurons in complex electromagnetic environments. Considering the memristive autapse involving magnetic coupling has voltagecontrolled, nonlinear, and memory, a 5-D HR neuron model containing magnetic field and electric field variables is established. Detailedly, the existence and stability conditions of the equilibrium point are determined by theoretical analysis, and the complex time-varying stability, saddle-node bifurcation, and Hopf bifurcation behaviors of the model are verified by numerical calculation. Interestingly, the system has a bistable structure consisting of quiescent state and period- 1 and period-2 bursting modes near the subcritical Hopf bifurcation. It is noteworthy that the memristive autapse has a complex regulation mechanism for the bistable region so that three kinds of bistable coexisting structures and counterintuitive dynamic phenomena can be induced by appropriately adjusting the memristive autapse. Accordingly, the mechanism of positive feedback memristive autapse decreases its firing frequency, while negative feedback memristive autapse promotes its excitability was revealed by the fast-slow dynamic analysis. Extensive numerical results display that the system generally possesses period-adding bifurcation modes and comb-shaped chaotic structures. Furthermore, it is found that the firing modes and multistability regions of the system can be accurately predicted by analyzing the global dynamic behaviors of Hamilton energy. Importantly, it is verified that the unidirectional coupling controller involving energy is far more efficient and consumes less energy than electrical synaptic coupling in achieving complete synchronization with mismatched parameters.

Keywords: Memristive autapse; Bistable; Multistability; Hamilton energy; Synchronization

\section{Introduction}

Neurons with nonlinear characteristics are involved in complex dynamic behaviors, including electrical activities, bifurcation, synchronization, and so on, in the process of realizing physiological and pathological functions of the organism [1-3]. The electrical activities of neurons are inevitably influenced by excitatory and inhibitory external stimuli and the self-feedback regulation of autapse. Accordingly, leading to various types of firing modes, including quiescent state, spiking, bursting, and even mixed-mode oscillations (MMOs) [4,5]. This plays a very important role in understanding the mechanism of neural information processing and the global dynamic behaviors of the nervous system [6-8]. Therefore, it is very necessary to establish or improve all kinds of continuous or discrete neuron models to accurately predict the firing activities and transition patterns of real neurons [9-13]. Indeed, the mechanism regulating neuron firing activities is very complex. More important practical factors need to be considered except for the external stimulus current, such as

S. Qiao, C. H. Gao (Author for correspondence), X. Y. He, J. J. Wang

College of Mathematics and Statistics, Northwest Normal University, Lanzhou 730070, China

e-mail: gaokuguo@163.com

X. L. An

School of Mathematics and Physics, Lanzhou Jiaotong University, Lanzhou,730070, China

College of Electrical and Information Engineering, Lanzhou University of Technology, Lanzhou 730050, China 
the change of membrane potential caused by the electromagnetic induction field excited when the concentration of charged ions in the cell fluctuates [14]. It was demonstrated in Refs. [15,16] that the electrical activities of neurons can stimulate time-varying electromagnetic fields inside and outside the cell, meanwhile, the feedback modulation of magnetic flux on the membrane potential of traditional Hindmarsh-Rose (HR) neuron model has been realized based on memristor. It is found that memristive current and electromagnetic radiation can promote the adaptive behavior of neuronal models to external stimulus, resulting in multiple modes of electrical activities and complex migration rules. Wu et al. [17] found that induced current can regulate the electrical activities and spatial patterns evolution of the Fitzhugh-Nagumo (FHN) neuron model, while external electromagnetic radiation can effectively inhibit the formation of spiral waves. Bao et al. [18] characterized the induction current by a magnetic flux-controlled memristor with hyperbolictype and improved a three-dimensional M-HR neuron model without equilibrium points. Meanwhile, the hidden and coexisting firing behaviors of the model are verified by numerical and circuit simulations. Refs. $[19,20]$ confirmed that the HR neuron model exposed to the magnetic field has subcritical Hopf bifurcation behavior, hidden firing activities, and MMOs, etc., and its hidden dynamic behaviors are controlled based on the Washout controller. These results reveal that electromagnetic induction plays an important role in modulating the electrical activities of neurons and provides useful exploration for studying the abnormal firing of neurons.

The multistability or multi-attractor coexistence induced by systems with different initial states is one of the most interesting topics in nonlinear dynamics, it should be emphasized that the parameters of the system remain constant throughout the process. So far, there have been extensive reports on the multistability-related research results of oscillating circuit models [21-24], mechanical systems [25-27], neuron models [28-30], and other types of dynamical systems [31-34]. It is worth mentioning that these systems with multistability tend to be non-autonomous or have nonlinear function terms with periodic characteristics (such as sine or cosine function), resulting in the equilibrium points and the stability of the system are time-varying [28,29] or initial value dependence [35]. Furthermore, abundant results have been obtained in constructing systems with multistability by hyperbolic-type memristors. For example, a memristive FHN circuit model with linear stable equilibrium point was established in Ref. [36], and its extreme multistability and dynamic mechanism were revealed by numerical means. Ref. [37] proposed an improved threedimensional Hopfield neural network (HNN) based on hyperbolic memristor, and a large number of asymmetric coexisting attractors can be induced by different initial values. Ref. [38] has investigated the global multistability of an adapting synapse-based neuron model, interestingly, the stability of the equilibrium points of the system shows periodic switching by appropriate external stimuli. Chen et al. [39] established an HNN coupling model containing two neurons by a voltagecontrolled memristor and confirmed its coexistence bifurcation patterns through using the local basins of attraction and phase trajectory. These numerical results fully reveal the dynamic mechanism of the coexistence of multi-mode firing activities in the nervous.

Recently, it has been confirmed that synapses or inductive currents can cause a large number of paradoxical phenomena, including post-inhibitory rebound (PIR) and post-inhibitory facilitation (PIF) [40,41]. It is a common dynamic phenomenon that inhibitory stimuli promote the bursting activities or firing frequency of neurons. Li et al. [42] found that a class of delay-free inhibitory autapse can induce the bursting firing activities of the Chay neuron model to occur period-adding bifurcation behavior, which will lead to the increase of its firing frequency, meanwhile, the feedback 
mechanism is explained by the separation of fast-slow variables. Lu et al. [43] validated the firing patterns of the Morris-Lecar (ML) neuron model under the regulation of inhibitory autapse, the results indicated that the autapse increase rather than decrease the firing frequency by inducing the transformation of the three types of bursting modes with the enhancement of inhibitory feedback. Notably, Wu et al. [44] reported a new case in which the suppressed induced current can promote the firing activities of the HR neural model, whose typical characteristic is that the bursting modes present a period-adding bifurcation without chaos, resulting in more spikes per cluster. Moreover, inhibitory synapses or stimuli have been shown to promote synchronous and coherent resonance behaviors of neural networks through the PIR mechanism [45,46]. Conversely, Wang et al. [47] explored another counterintuitive neurodynamic phenomenology in detail, that is, a class of excitatory autapse attenuates rather than increases bursting activities by inducing the ML neuron model to produce reverse period-adding bifurcation behaviors. Ref. [48] found that positive induced current can inhibit the firing frequency of the HR neuron model, which depends crucially on the setting of internal parameters of the memristor. Similarly, Ref. [49] proposes a memristive synapse to mimic the properties of a biological synapse, the interesting finding is that the inhibitory synaptic current can promote the electrical activities of the neuron, while the excitatory synaptic current can inhibit its electrical activities. Inspired by this, we optimize the internal parameters of the memristive synapse to make it have global uncommon dynamic features and further clarify the complex feedback mechanism of the synapse to the bistable regions.

It is well known that the processing and transmission of neural information depend on the supply and consumption of energy, so it is very important to understand the energy changes related to electrical activity. Wang et al. [50] confirmed that the energy evolution of the HR neuron model highly relies on its electrical activities regulated by external stimuli. The results show that energy needs to be consumed during the transition from spiking to bursting state, while the transition from bursting to spiking states is the process of energy release. Yang et al. [51] showed that the amplitude of external electromagnetic radiation had a greater impact on the energy of the Izhikevich neuron than its frequency. In particular, the high-frequency electrical activities predict a lower average energy because each action potential contains a certain amount of energy [51,52]. Moreover, the complex exchange of internal energy can be realized and the chaotic oscillation behaviors can be effectively inhibited by applying appropriate energy feedback to systems [53-55]. However, more importantly, there are no reports on locating the multi-attractor coexisting regions and realizing the synchronization with mismatched parameters by calculating and determining the global dynamic behaviors of the energy function, so this is also the focus of the paper.

The rest of the paper is organized as follows. In Sect. 2, the HR neuron model with memristor synapses under the action of the electric field is established, and the numerical tools and algorithms involved in this study are introduced in detail. In Sect. 3, the time-varying stability and global bifurcation behaviors of the equilibrium points of the model are analyzed, and the firing modes with bistable features are simulated. In Sect. 4, the regulation of the memristive autapse induction to bistable regions is studied, and the mechanism of its counterintuitive firing behaviors is revealed by fast-slow dynamic analysis. In Sect. 5, it is proved that the Hamiltonian energy of the system can effectively reflect the coexisting regions of multiple attractors and realize synchronization with unidirectional coupling involving energy. The relevant conclusions are summarized in Sect. 6.

\section{Theoretical Model and methods}




\subsection{Model description}

Generally, it will cause magnetization and polarization of the medium when the systems are exposed to an external electromagnetic field, which will lead to changes in the distribution and transmission of various charged ions (e.g., potassium, sodium, and calcium ions) inside and outside the cell, meanwhile, inducing time-varying induced electric field and current [56,57]. Ma et al. [58] realized the polarization modulation of media caused by external electric field changes by introducing electric field variables and confirmed that different electric fields could lead to complex electrical activities and evolution modes in the FHN neuron model. Ref. [59] further explored the basins of attraction for the model, and the results showed that the FHN neuron model does not have multistability under an electric field. Nevertheless, the HR neuron model can be induced to produce rich multi-attractor coexistence phenomena under an external periodic electric field [30,60]. According to Maxwell's electromagnetic field theory, a time-varying magnetic field can generate an induced electric field and vice versa. Accordingly, a reliable neuron model under electromagnetic induction should consider the interaction of induced electric field and magnetic field together [61]. Moreover, considering memristive autapse involving magnetic coupling has many features similar to electromagnetic induction current, such as voltage-controlled, nonlinear, and memory $[49,62]$. Thus, the memristive autapse and electric field variables are simultaneously introduced into the classical HR neuron model, and it is described as

$$
\left\{\begin{array}{l}
\&=y-a x^{3}+b x^{2}-z+I+I_{M}, \\
\&=c-d x^{2}-y+k_{0} E, \\
\&=r\left[s\left(x-\chi_{0}\right)-z\right], \\
\&=k_{1} y-k_{2} E+E_{e x t} .
\end{array}\right.
$$

where $x, y, z$, and $E$ represent membrane voltage, recovery variable of fast current related to potassium and sodium ions, adaptive-slow current, and electric field, respectively. Note that the variable $k_{0} E$ is applied to fast variable $y$ instead of slow variable $z$ to describe the modulation effect of induced electric field due to the fast current is more sensitive to changes in polarized and induced electric fields [57-60]. The feedback variable $-k_{5} E$ denotes the leakage electric field generated by the adaptive adjustment of neurons to the electric field $E[30,61]$, which enables the system to have better stability. $I$ is external stimulus current. $a, b, c, d, r, s$, and $\chi_{0}$ are kinetic constants related to the ionic current and conductance of the model. $E_{e x t}$ shows periodic disturbance or random noise in the external electric field [58], thus without loss of generality, $E_{e x t}=A \cos (2 \pi f t)$ is selected, where the parameters $A$ and $f$ represent the amplitude and frequency of the periodic disturbance, respectively. Furthermore, $I_{M}$ denotes the output current through the memristive autapse involving magnetic coupling, and to reflect its memory and plasticity, the expression involved in Refs. $[49,62]$ is

$$
\left\{\begin{array}{l}
I_{M}=g_{M} w^{2}\left(V_{e}-x\right), \\
L_{\&}=k_{3} x-k_{4} w .
\end{array}\right.
$$

where $w$ is the variable of the magnetic field after dimensionless processing. $g_{M}$ represents the feedback intensity of the memristive autaptic current. $V_{e}, k_{3}$, and $k_{4}$ are dimensionless parameters. Importantly, the parameters $V_{e}=0, k_{3}=0.3$, and $k_{4}=0.4$ were selected to achieve the memory features between the autaptic current $I_{M}$ and the membrane voltage $x$. Thus, it may be assumed that the input voltage for Eq. (2) is $x=v=V_{M} \sin \left(2 \pi f_{0} t\right)$. As is intuitively depicted in Figs. 1(a)- 
(c), the numerical relationship between memristor synaptic current $I_{M}$ and voltage $v$ presents the characteristics of pinched hysteresis loops under the combination of different parameters $V_{M}$, $f_{0}$, and $V_{e}$. It is observed that the $I_{M}-v$ curves in Figs. 1(a) and (b) have two closed loops. Particularly, when $V_{e} \neq 0$, the $I_{M}-v$ curves in Fig. 1(c) have three closed loops. These numerical results fully demonstrate the memory and plasticity of the memristive autapse.

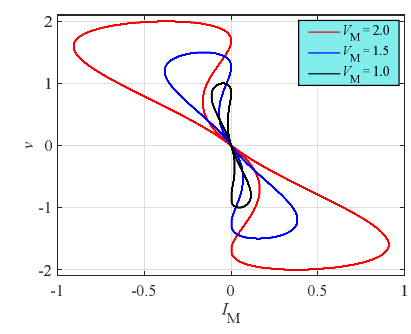

(a)

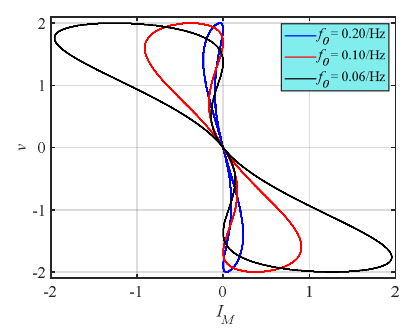

(b)

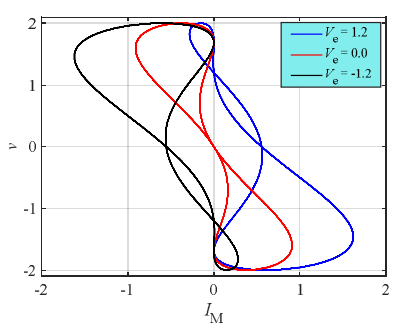

(c)

Fig. 1 Numerically simulated pinched hysteresis loops of the memristive autapse on the $I_{M}-v$ plane. (a) $\left(f_{0}, V_{e}\right)=(0.1,0) ;(\mathrm{c})\left(V_{M}, V_{e}\right)=(2,0) ;(\mathrm{c})\left(f_{0}, V_{M}\right)=(0.1,2)$.

\section{2 methods}

The reference parameter values of the system (1) are selected as $a=1.0, b=3.0, c=1.0$, $d=5.0, s=4.0, r=0.006, \chi_{0}=-1.61, k_{0}=0.1, g_{M}=0.1, V_{e}=0, k_{1}=0.2, k_{2}=0.5, k_{3}=0.3$, $k_{4}=0.4, I=3.0, A=0$, and $f=0.01$. Corresponding numerical simulation diagrams were obtained by MATCONT package, XPPAUTO software, and parallel computing [63-65], including stability distribution diagrams, two-parameter bifurcation diagrams, and the maximum Lyapunov exponent diagrams, etc. The fourth-order Runge-Kutta algorithm with step size 0.01 and initial value fixed at $(0.1,0.1,0.1,0.1,0.1)$ is applied in numerical calculation and process.

\section{Global stability analysis and bistable}

\subsection{Time-varying stability and bifurcation}

Obviously, the relevant information about equilibrium points of system (1) can be obtained by solving the equations $\&=\&=0, \&=0$, which is described by

$$
\left\{\begin{array}{l}
y-a x^{3}+b x^{2}-z+I_{e x t}+g_{M} w^{2}\left(V_{e}-x\right)=0, \\
y=c-d x^{2}+k_{0} E \\
z=s\left(x-\chi_{0}\right) \\
E=\left(k_{1} y+E_{e x t}\right) / k_{2}, \\
w=k_{3} x / k_{4} .
\end{array}\right.
$$

The real solution $\left(x_{e}, y_{e}, z_{e}, E_{e}, w_{e}\right)$ of Eq. (3) is the equilibrium point $P_{e}=\left(x_{e}, y_{e}, z_{e}, E_{e}, w_{e}\right)$ of the system (1). To facilitate the analysis of the existence of solution of Eq. (3), the cubic equation $F(x)$ about variable $x$ can be obtained by simplified Eq. (3), which is expressed as

$$
F(x)=F_{0} x^{3}+F_{1} x^{2}+F_{2} x+F_{3}=0
$$

where 


$$
\left\{\begin{array}{l}
F_{0}=-\left(a+g_{M} k_{3}^{2} / k_{4}^{2}\right), \\
F_{1}=b-d k_{2} /\left(k_{2}-k_{0} k_{1}\right)+g_{M} V_{e} k_{3}^{2} / k_{4}^{2}, \\
F_{2}=-s, \\
F_{3}=k_{2}\left(c+k_{0} E_{e x t} / k_{2}\right) /\left(k_{2}-k_{0} k_{1}\right)+s \chi_{0}+I_{e x t} .
\end{array}\right.
$$

Let $A_{1}=F_{1}^{2}-3 F_{0} F_{2}, A_{2}=F_{1} F_{2}-9 F_{0} F_{3}$, and $A_{3}=F_{2}^{2}-3 F_{1} F_{3}$, so the total discriminant is $\Delta=A_{2}^{2}-4 A_{1} A_{3}$. According to Shengjin's formula, the existence of equilibrium points of system (1) can be seen in the following three situations.

If $\Delta>0$, then the system (1) has only one equilibrium point $P_{e}=\left(x_{e}, y_{e}, z_{e}, E_{e}, w_{e}\right)$, which is expressed as

$$
\left\{\begin{array}{l}
x_{e}=\left(-F_{1}-\left(\sqrt[3]{X_{1}}+\sqrt[3]{X_{2}}\right)\right) \\
y_{e}=k_{2}\left(c-d x_{e}^{2}+k_{0} E_{e x t} / k_{2}\right) /\left(k_{2}-k_{0} k_{1}\right), \\
z_{e}=s\left(x_{e}-\chi_{0}\right) \\
E_{e}=\left(k_{1} y_{e}+E_{e x t}\right) / k_{2} \\
w_{e}=k_{3} x_{e} / k_{4}
\end{array}\right.
$$

where $X_{1,2}=A_{1} F_{1}+3 F_{0}\left(-A_{2} \pm \sqrt{A_{2}^{2}-4 A_{1} A_{3}}\right) / 2$.

If $\Delta=0$, then the system (1) has two equilibrium points $P_{e_{i}}=\left(x_{e_{i}}, y_{e_{i}}, z_{e_{i}}, E_{e_{i}}, w_{e_{i}}\right),(i=1,2)$, which are expressed as

$$
\left\{\begin{array}{l}
x_{e_{1}}=-F_{1} / F_{0}+Y, x_{e_{2}}=-Y / 2_{0}, \\
y_{e_{i}}=k_{2}\left(c-d x_{e_{i}}^{2}+k_{0} E_{e x t} / k_{2}\right) /\left(k_{2}-k_{0} k_{1}\right), \\
z_{e_{e_{i}}}=s\left(x_{e_{i}}-\chi_{0}\right) \\
E_{e_{i}}=\left(k_{1} y_{e_{i}}+E_{e x t}\right) / k_{2} \\
w_{e_{i}}=k_{3} x_{e_{i}} / k_{4}
\end{array}\right.
$$

where $Y=A_{2} / A_{1},\left(A_{1} \neq 0\right)$.

If $\Delta<0$, then the system (1) has three equilibrium points $P_{e_{i}}=\left(x_{e_{i}}, y_{e_{i}}, z_{e_{i}}, E_{e_{i}}, w_{e_{i}}\right),(i=1,2,3)$, which are expressed as

$$
\left\{\begin{array}{l}
x_{e_{i}}=\left(-F_{1}-2 \sqrt{A_{1}} \cos ((2 \pi i+\theta) / 3)\right) / 3 F_{0}, \\
y_{e_{i}}=k_{2}\left(c-d x_{e_{i}}^{2}+k_{0} E_{e x t} / k_{2}\right) /\left(k_{2}-k_{0} k_{1}\right), \\
z_{e_{i}}=s\left(x_{e_{i}}-\chi_{0}\right) \\
E_{e_{i}}=\left(k_{1} y_{e_{i}}+E_{e x t}\right) / k_{2}, \\
w_{e_{i}}=k_{3} x_{e_{i}} / k_{4} .
\end{array}\right.
$$

where $\theta=\arccos T, T=\left(2 A_{1} F_{1}-3 F_{0} A_{2}\right) / 2 \sqrt{A_{1}^{3}}, A_{1}>0,-1<T<1$.

Moreover, the stability of system (1) at the equilibrium point $P_{e}=\left(x_{e}, y_{e}, z_{e}, E_{e}, w_{e}\right)$ is determined by the corresponding Jacobian matrix, which is described as 


$$
J=\left[\begin{array}{ccccc}
-3 a x_{e}^{2}+2 b x_{e}-g_{M} w_{e}^{2} & 1 & -1 & 0 & 2 g_{M} w_{e}\left(V_{e}-x_{e}\right) \\
-2 d x_{e} & -1 & 0 & k_{0} & 0 \\
r s & 0 & -r & 0 & 0 \\
0 & k_{1} & 0 & -k_{2} & 0 \\
k_{3} & 0 & 0 & 0 & -k_{4}
\end{array}\right]
$$

Noted that Eqs. (4)-(7) contain the periodic disturbance term $E_{e x t}=A \cos (2 \pi f t)$ of the external electric field. Accordingly, it can be known that the equilibrium point $P_{e}$ depends on internal parameters and time $t$, which will lead to the time-varying equilibrium state and stability of the system (1). For simplicity, when $I=1.3$ and other parameters take reference values, there is discriminant $\Delta>0$, that is, the system (1) only has an equilibrium point. Meanwhile, it is necessary to focus on the evolution law of the time-varying stability of the system (1) concerning parameters $A, k_{0}$, and $g_{M}$. Figs. 2(a)-(i) show that the stability or type of equilibrium point of the system (1) presents a spatiotemporal variation pattern of symmetry and periodicity, where different color areas represent diverse types of equilibrium points. When $k_{0}=0.1$, Fig. 2(a) displays that system (1) has equilibrium points of two types on the $t \in[0,400], A \in[-3,3]$ plane. It can be seen that stable focal points and unstable focal points coexist, and the two kinds of equilibrium points realize mutual transformation via Hopf bifurcation behavior. Interestingly, as parameter $k_{0}$ gradually increases, the system (1) will have more complex time-varying stability. When $k_{0}=0.2$, new unstable nodes appear in the previously unstable focal point regions, resulting in three time-varying equilibrium points in the system (1), among which a stable focal point coexists with two unstable equilibrium points, as shown in Fig. 2(b). When $k_{0}=0.3$, new stable nodes appear in the previously stable focal point regions, resulting in four time-varying equilibrium points in the system (1), in which two stable points coexists with two unstable equilibrium points, as shown in Fig. 2(c). Furthermore, note that as parameter $k_{0}$ increases, the region of stable equilibrium (light blue and green regions) becomes smaller, while the unstable equilibrium region (red and yellow regions) becomes larger. Importantly, new stable (or unstable) regions can only evolve within the original stable (or unstable) regions. Fig. 2(d) shows that system (1) has three timevarying equilibrium points on the $t \in[0,400], k_{0} \in[-3,3]$ plane when $A=1$, among which two stable equilibrium points coexist with one unstable equilibrium point. The results in Fig. 2(e) showed that new unstable focal points appear in the regions of previously stable focal point, and new unstable nodes appear in the regions of previously unstable focal point when $A=2$, resulting in four time-varying equilibrium points in the model. When $A=3$, Fig. 2(f) displays that the unstable equilibrium points regions expand significantly, while no new equilibrium point is generated. Similarly, Figs. 2(g)-(i) exhibit the system (1) has four time-varying equilibrium points on the $t \in[0,400], g_{M} \in[-1,1]$ plane. Notably, parameter $A$ cannot change the coexistence type of equilibrium points, but it can effectively expand the regions of unstable equilibrium points. These numerical results confirmed that periodic external electric field disturbances can lead to complex time-varying stability of the system (1), meanwhile, the time-varying equilibrium points usually 
predict the initial value sensitivity and multistability of systems [28,29].

To fully understand the global stability distribution features and bifurcation patterns of the system (1) in different parameter spaces, it is necessary to limit the time-varying stability of the system (i.e., let $A=0$ ). Based on the MATCONT package [63], the existence of equilibrium points and bifurcation laws of the system (1) on different parameter planes can be obtained efficiently, as shown in Figs. 3(a)-(c). Note that diverse color areas represent different conditions of the existence and stability of equilibrium points. More precisely, the system (1) has only one equilibrium point in the $\mathrm{i}-\mathrm{i}(\mathrm{i}=1,2,3$, and 4$)$ regions, while the system has three equilibrium points in the II- $\mathrm{i}(\mathrm{i}=1,2$, and 3 ) regions. That is, the I-1 region represents stable nodes, the I-2 region indicates the stable focal points, the I-3 region denotes the unstable focal points, the I-4 region describes unstable nodes, the II-1 region represents the coexistence of three unstable equilibrium points, the II- 2 region denotes the coexistence of a stable equilibrium point and two unstable equilibrium points, and the II-3 region represents the coexistence of two stable equilibrium points and an unstable equilibrium point. Moreover, the red curves denote Hopf bifurcation behaviors, and the black curves indicate saddle-node bifurcation behaviors. Fig. 3(a) confirms that there are seven cases of the existence and stability of equilibrium point of system (1) on the $I \in[1,6], k_{0} \in[-0.5,1.0]$ plane, and the I-i (i $=1,2,3$, and 4$)$ and II-j $(j=1,2)$ regions are in contact via saddle-node bifurcation. Meanwhile, there are three Hopf curves in the parameter plane, which indicates that the model has a complex firing mechanism. In other words, generally, neurons change the stability of their equilibrium point from a stable state to an unstable state by Hopf bifurcation, resulting in generate corresponding limit cycle attractors or various types of electrical activities. Similarly, Figs. 3(b) and (c) also reveal that system (1) has complex stability distribution and saddle-node bifurcation and Hopf bifurcation behaviors. Furthermore, the relationship between the stability of equilibrium and the electrical activities of the system is worth further investigation.

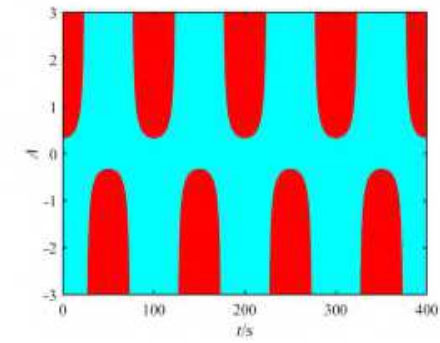

(a)

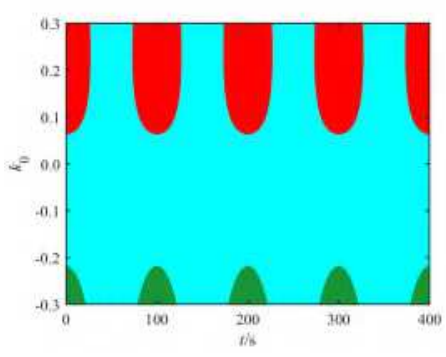

(d)

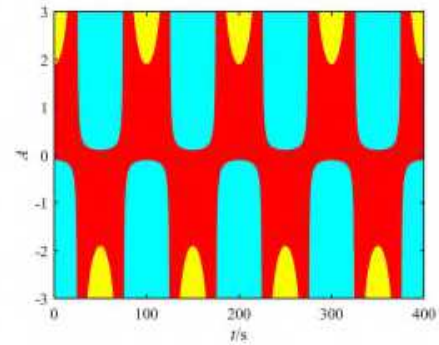

(b)

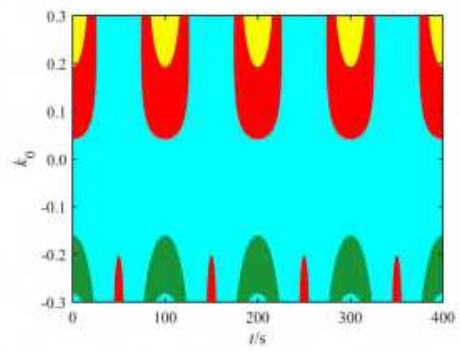

(e)

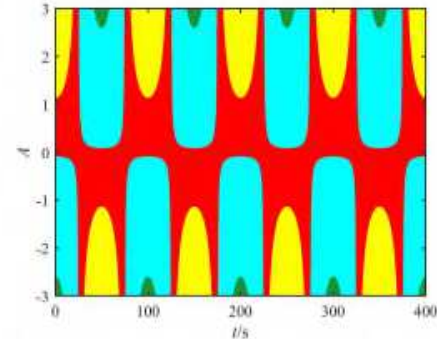

(c)

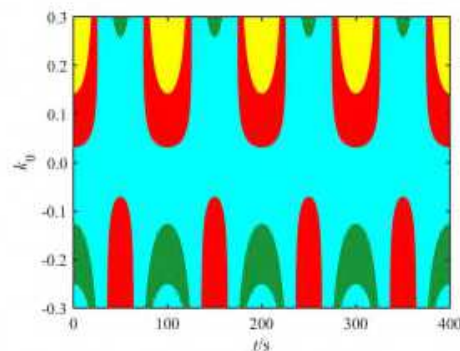

(f) 


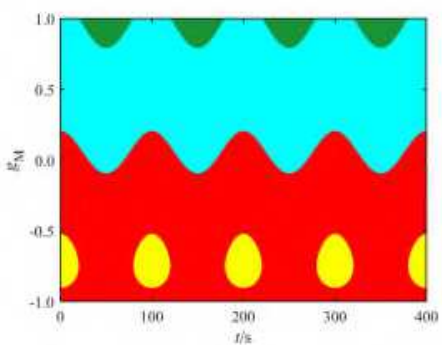

(g)

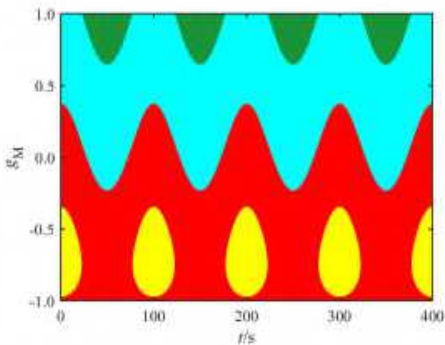

(h)

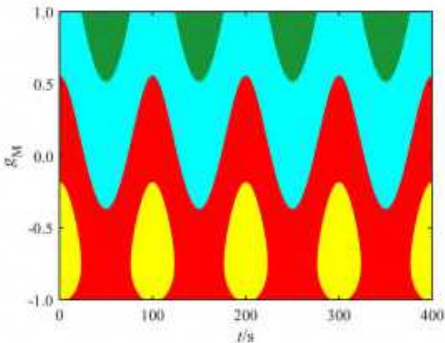

(i)

Fig. 2 Time-varying stability distribution diagrams of the system (1). The green area represents the stable nodes, the blue area describes the stable focal points, the red area denotes the unstable focal points, and the yellow area shows unstable nodes. (a) $k_{0}=0.1$; (b) $k_{0}=0.2$; (c) $k_{0}=0.3$; (d) $A=1$; (e) $A=2$; (f) $A=3$, (h) $A=1$; (g) $A=2$; (i) $A=3$.

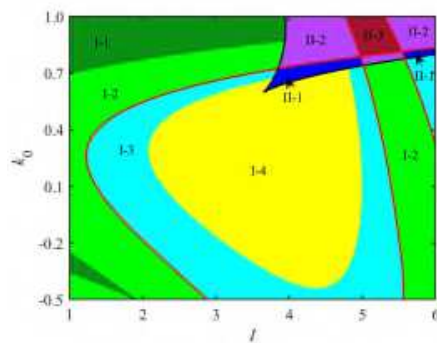

(a)

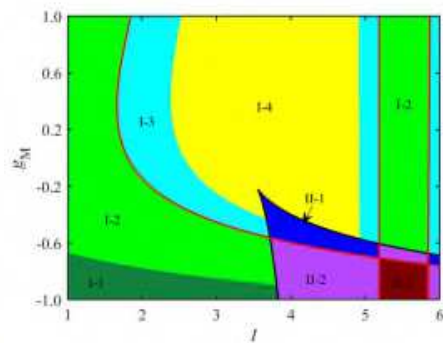

(b)

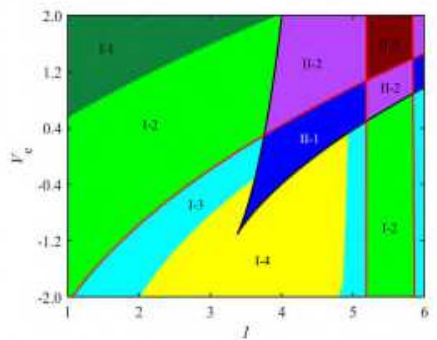

(c)

Fig. 3 Stability distribution of equilibrium points and its Hopf bifurcation and saddle-node bifurcation behaviors of the system (1) on different parameter planes. (a) $I \in[1,6], k_{0} \in[-0.5,1],\left(g_{M}, V_{e}\right)=(0.1,0)$; (b) $I \in[1,6], g_{M} \in[-1,1]$, $\left(k_{0}, V_{e}\right)=(0.5,0) ;(\mathrm{c}) I \in[1,6], V_{e} \in[-2,2],\left(k_{0}, g_{M}\right)=(0.5,-0.5)$.

\subsection{Bistable and coexisting firing modes}

It is noteworthy that an unstable equilibrium point can stimulate various firing activities, while a stable equilibrium point induces a quiescent state according to the traditional view of neuron dynamics. In particular, there is another firing mechanism in the neuron model [30], that is, the model with a stable equilibrium point in the vicinity of subcritical Hopf bifurcation can produce various hidden firing modes by appropriate adjustment of initial values, which is a common bistable phenomenon. To determine the bistable structure of the system (1) and further study the regulation of memristive autaptic current $I_{M}$, let parameters $k_{0}=0.12$ and $g_{M}=0$. Fig. 4 exhibits the global basin of attraction and Hopf bifurcation behavior of the system (1) on the $I \in[1.1,1.3]$, $x(0) \in[-20,20]$ plane based on the MATCINT package [63], where different firing patterns are distinguished by corresponding color areas. Note that the remaining initial values $y(0), z(0)$, $E(0)$, and $w(0)$ remain at the corresponding equilibrium point in the numerical simulation, thus these initial values will change as the parameter $I$ changes. It can be seen that the firing activities of the system near Hopf not only depend on the external stimulus current $I$ but also the initial value $x(0)$ plays a decisive role. Importantly, MATCONT software can efficiently calculate the corresponding numerical results of the system (1) at the Hopf as follows: The external stimulus current is $I=I_{H}=1.2024114$, the equilibrium point is $P_{H}=(-1.46927723,-10.28768688$, $0.56289108,-4.11507475,-1.10195792)$, the corresponding eigenvalues are $\lambda_{1,2}^{H}= \pm 0.03529726 i$, 
$\lambda_{3}^{H}=-0.54416223, \lambda_{4}^{H}=-16.25382792, \lambda_{5}^{H}=-0.40$, and the first Lyapunov coefficient is $L=0.00034876>0$. It indicates that the subcritical Hopf bifurcation occurs in the system (1), and the bifurcation direction is the direction in which the external stimulus current $I$ decreases. To understand the initial value sensitivity of the system (1) in detail, it is necessary to further study the firing activities near the Hopf bifurcation.

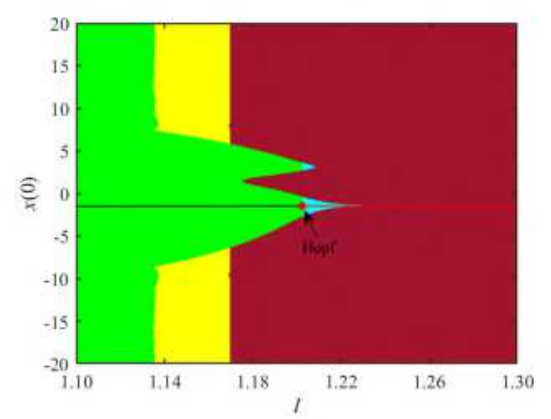

Fig. 4 Global basins of attraction of the system (1) on the $I \in[1.1,1.3], x(0) \in[-20,20]$ plane. The green area represents the quiescent state; The red area denotes the period-2 bursting; The yellow area indicates the period-1 spiking; The light blue area describes the subthreshold oscillations with increasing amplitude; The black curve shows stable node-focus; The red curve shows unstable node-focus; The red solid point is the Hopf bifurcation point.

Fig. 4 displays that system (1) has an unstable equilibrium point and is in the coexistence state of subthreshold oscillations with increasing amplitude and period-2 bursting when $I_{H}<I \leq I^{*}=1.2241$. Specifically, when $I_{1}=1.21 \in\left(I_{H}, I^{*}\right]$, the equilibrium point is $P_{1}=(-$ $1.46730830,-10.25731952,0.57076681,-4.10292781,-1.10048122)$, and the corresponding eigenvalues are $\lambda_{1,2}^{1}=0.00023428 \pm 0.03529036 i, \lambda_{3}^{1}=-0.54411410, \lambda_{4}^{1}=-16.22518515$, and $\lambda_{5}^{1}=-0.40$, so the equilibrium point $P_{1}$ is an unstable node-focus. Note that the equilibrium point can excite the subthreshold oscillations with divergent features, but it cannot induce the model produce firing activities. The time response diagram and phase trajectory are depicted in Fig. 5(a) and (b), respectively. However, when the initial value is $q_{1}=(-1.376,-8.672,0.602,-4.103,-1.101)$ near the equilibrium point $P_{1}$, the system (1) goes through the subthreshold oscillations with a duration of about 1000/s (as shown in the black curve in Fig. 5(c)), then an unstable period-1 spiking is generated (as shown in the green curve in Fig. 5(c)), and finally the stable period-2 bursting state (as shown in the blue curve in Fig. 5(c)). Correspondingly, the phase trajectory is shown in Figs. 5(d) and (e). Meanwhile, Fig. 5(f) exhibits the basin of attraction of the system (1) on the $z(0)-x(0)$ plane. It is observed that the region at the upper left corner of the unstable equilibrium point $P_{1}$ can also induce the system (1) to produce subthreshold oscillations. This indicates that the unstable equilibrium points near Hopf bifurcation may not excite the firing activities of neurons, which crucially relies on the selection of initial value. 


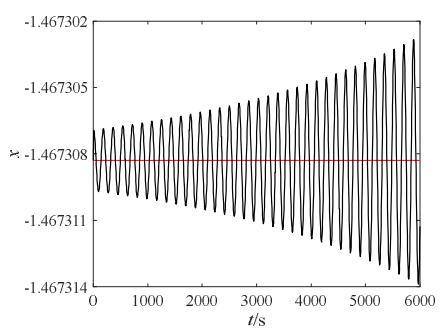

(a)

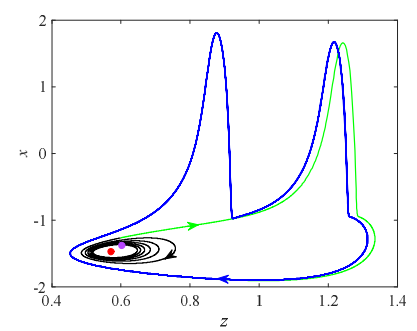

(d)

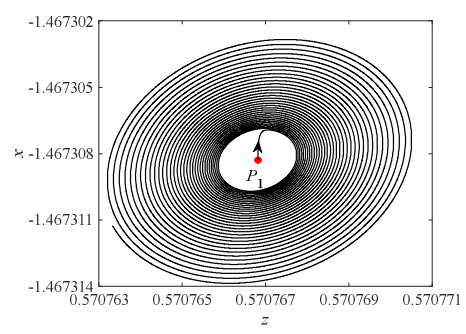

(b)

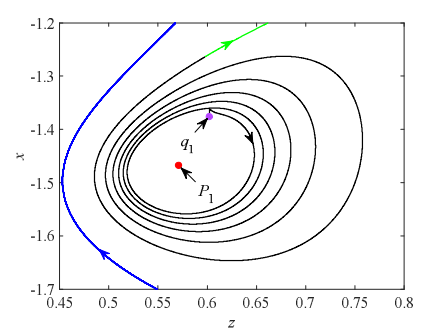

(e)

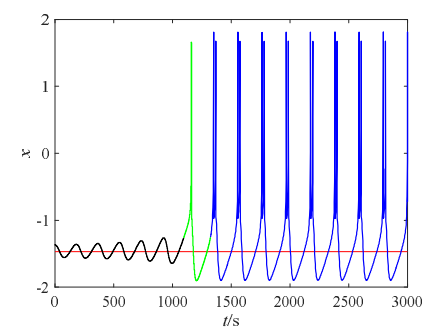

(c)

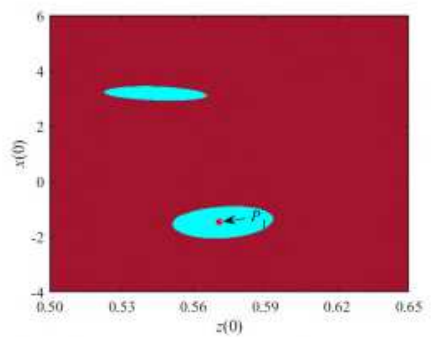

(f)

Fig. 5 Time response diagrams, phase trajectories and basin of attraction of the system (1) at different initial values when $I=I_{1}$. The blue curve shows the period-2 bursting. The black curve represents the subthreshold oscillations with increasing amplitude. The green curve is the unstable firing mode. The red line is the membrane voltage $x$ at the unstable equilibrium point $P_{1}$; The red dot is unstable equilibrium $P_{1}$. (a) The subthreshold oscillations with increasing amplitude by unstable equilibrium $P_{1}$; (b) Phase trajectory; (c) The period-2 bursting state excited by initial value $q_{1}$; (d) Phase trajectory; (e) Enlargement of Fig. (d); (f) The basin of attraction of equilibrium point $P_{1}$, note that the corresponding meaning of its color regions is consistent with that in Fig. 3.

When parameter $I$ gradually decreases to $I_{H}$, subcritical Hopf behavior occurs in the system (1), which indicates that the unstable equilibrium point will change to a stable equilibrium point and an unstable limit cycle will be generated. Notably, Fig. 4 confirms that the system (1) has two types of bistable structures after Hopf bifurcation occurs, that is, the quiescent state coexists with the period-1 and period-2 bursting. For $I_{0}=1.1692 \leq I<I_{H}$, system (1) is in the coexistence state of the quiescent state and period-2 bursting, which mainly depends on the distance between the initial value and the equilibrium point. When $I_{2}=0.18 \in\left[I_{*}^{0}, I_{\mathrm{H}}\right)$, the equilibrium point is $P_{2}$ $=(-1.47506691,-10.37721849,0.53973235,-4.15088740,-1.10630019)$, and the eigenvalues are $\lambda_{1,2}^{2}=-0.00068751 \pm 0.03530888 i, \lambda_{3}^{2}=-0.54430387, \lambda_{4}^{2}=-16.33818978$, and $\lambda_{5}^{2}=-0.40$, so the equilibrium point $P_{2}$ is the stable node-focus. When the initial value is $q_{2}=(-1.475,-10.377$, $0.540,-4.151,-1.106$ ), the results in Figs. 6(a) and (b) display that system (1) finally converges to the equilibrium point $P_{2}$. Furthermore, when the value of parameter $I_{2}$ remains unchanged and the initial value is $q_{3}=(-1.475,-6.986,0.626,-4.151,-1.106)$, system (1) can produce period-2 bursting, and its time response diagram and phase trajectory are shown in Fig.6 (c)-(e), respectively. It can be seen that system (1) goes through the subthreshold oscillations with a duration of about 1100/s (as shown by the black curve in Fig. 6(c)), then generates an unstable firing pulse of period1 and period-2 (as shown by the green curve in Fig. 6(c)), and finally enters a stable period-2 bursting mode (as shown by the blue curve in Fig. 6(c)). Moreover, considering that equilibrium 
point $P_{2}$ is stable, the firing activities belong to the hidden mode [30], and its corresponding basin of attraction is depicted in Fig. 6(f). Note that this is different from unstable equilibrium $P_{1}$, whereas the basin of attraction induced by equilibrium $P_{2}$ is a simply connected region.

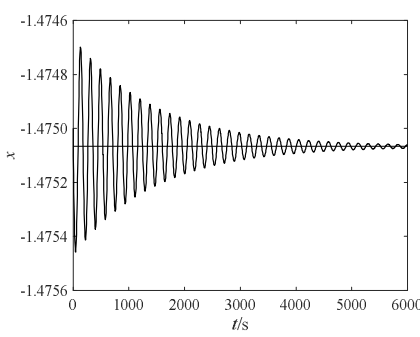

(a)

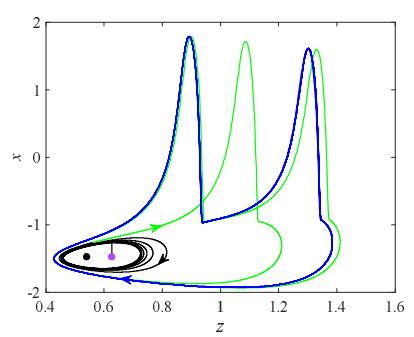

(d)

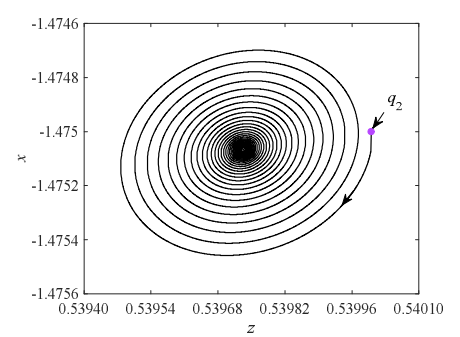

(b)

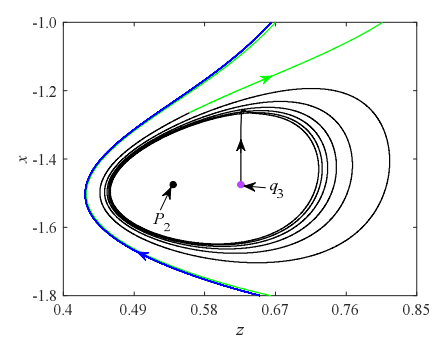

(e)

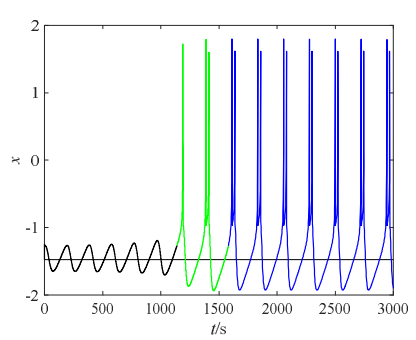

(c)

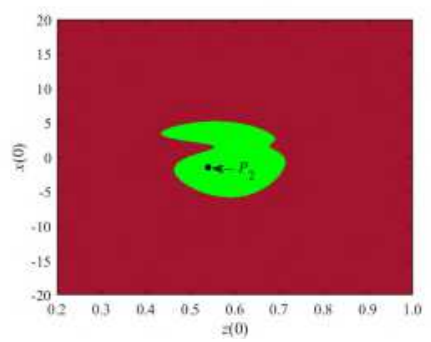

(f)

Fig. 6 Time response diagrams, phase trajectories and basin of attraction of the system (1) at different initial values when $I=I_{2}$. The blue curve represents the period-2 bursting. The black curve denotes the subthreshold oscillations with convergence trends. The green curve shows an unstable firing mode. The black line is the membrane voltage $x$ at the stable equilibrium point $P_{2}$. The black dot is a stable equilibrium $P_{2}$. (a) The quiescent state induced by stable equilibrium point $P_{2}$; (b) Phase trajectory; (c) The period-2 bursting state excited by initial value $q_{3}$; (d) Phase trajectory; (e) Enlargement of Fig. (d); (f) The basin of attraction of equilibrium point $P_{2}$, note that the corresponding meaning of its color regions is consistent with that in Fig. 3.

For $I_{*}=1.1343 \leq I<I_{0}$, Fig. 4 shows that system (1) has a bistable structure composed of quiescent state and period-1 spiking. When $I_{3}=0.14 \in\left[I_{*}, I_{0}\right)$, the equilibrium point is $P_{3}=(-$ $1.48530802,-10.53644913,0.49876792,-4.21457965,-1.11398101)$, and the corresponding eigenvalues are $\lambda_{1,2}^{3}=-0.00189865 \pm 0.03529799 i, \lambda_{3}^{3}=-0.54455496, \lambda_{4}^{3}=-16.48791560$, and $\lambda_{5}^{3}=-0.40$, which shows that the equilibrium point $P_{3}$ is also a stable node-focus. When the initial value is $q_{4}=(-1.485,-10.536,0.498,-4.214,-1.114)$, it is found in Figs. 7(a) and (b) that system (1) finally converge to the stable equilibrium point $P_{3}$, and the convergence speed of its membrane voltage $x$ is significantly accelerated compared with Fig. 6(a). In particular, when the parameter $I_{3}$ is fixed and the initial value is $q_{5}=(-1.581,-6.855,0.522,-4.215,-1.114)$, the system (1) can generate hidden period-1 spiking mode. Meanwhile, the time response diagram and phase trajectory are shown in Fig. 7(c)-(e), respectively. It can be seen that the system (1) goes through the subthreshold oscillations with a duration of about 1000/s (as shown by the black curve in Fig. 7(c)), then generates an unstable period-1 firing pulse (as shown by the green curve in Fig. 7(c)), and finally enters a stable period-1 spiking mode (as shown by the blue curve in Fig. 7(c)). Furthermore, Fig. 7(f) shows the basin of attraction of system (1) at equilibrium point $P_{3}$, it is found 
that the quiescent state region induced by equilibrium point $P_{3}$ is significantly larger than that of equilibrium point $P_{2}$, while its corresponding hidden firing region is smaller. These numerical results once again confirm that system (1) near Hopf bifurcation has complex firing mechanisms and hidden firing modes, which poses a challenge to distinguish the firing states of neurons through local dynamic analysis. More importantly, the evolution of the bistable structure under the action of memristive autapse is worth further discussed.

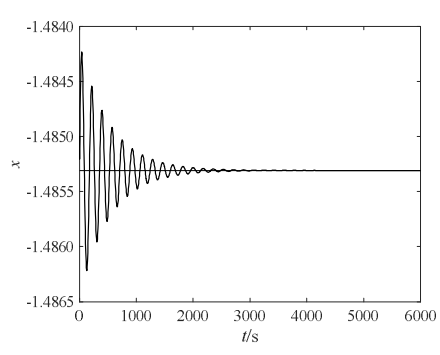

(a)

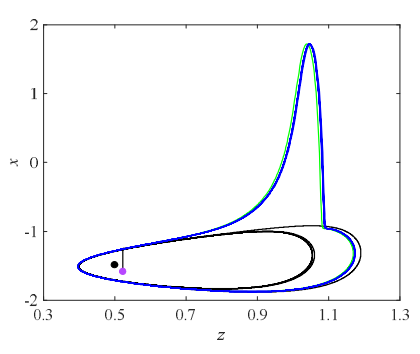

(d)

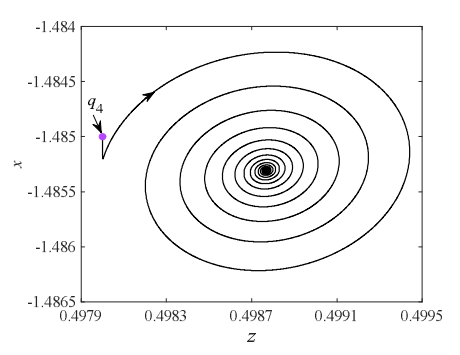

(b)

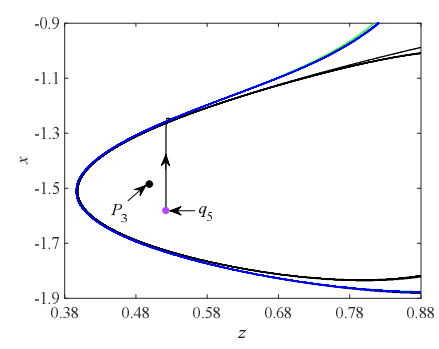

(e)

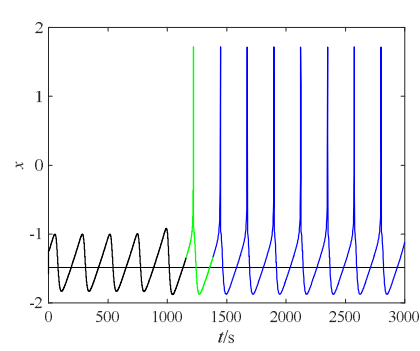

(c)

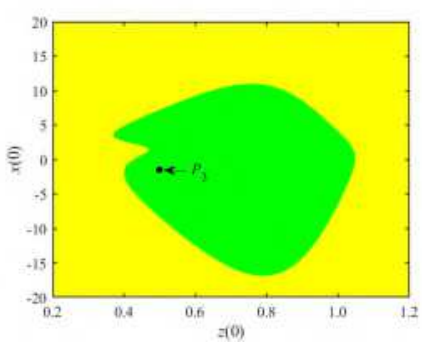

(f)

Fig. 7 Time response diagrams, phase trajectories and basin of attraction of the system (1) at different initial values when $I=I_{3}$. The blue curve shows the period-1 spiking. The black curve represents the subthreshold oscillations with convergence trends. The green curve denotes unstable discharge mode. The black line is the membrane voltage $x$ at stable equilibrium point $P_{3}$; The black dot is stable equilibrium points $P_{3}$. (a) The quiescent state induced by stable equilibrium point $P_{3}$; (b) Phase trajectory; (c) The period-1 spiking excited by initial value $q_{5}$; (d) Phase trajectory; (e) Enlargement of Fig. (d); (f) The basin of attraction of equilibrium $P_{3}$, note that the corresponding meaning of its color regions is consistent with that in Fig. 3.

\section{Bistable and counterintuitive dynamics induced by memristive autapse}

\subsection{Bistable and excitatory transitions by memristive autapse}

The above numerical results show that two types of bistable structures can be induced by external stimulus current and electric field in the absence of memristive autaptic current $I_{M}$. Similar to electrical autapse, there are positive and negative feedback types in the memristive autapse, that is, for $g_{M}>0, I_{M}$ represents the positive feedback memristive autapse current, while $I_{M}$ indicates the negative feedback memristive autapse current when $g_{M}<0$. To understand the mechanism of autaptic current $I_{M}$ to bistable and excitability of the system (1). When $V_{e}=0$ and the other parameters take reference values, Figs. 8(a)-(f) exhibit the evolution results of global basins of attraction under the action of different types and intensities of memristive autaptic current $I_{M}$. More precisely, Fig. 8(a) shows that subcritical Hopf bifurcation behavior occurs in the system (1) at parameter $I_{H}^{01}=1.338146$ when $g_{M}=0.1$. It is observed that the 
stability of the model is enhanced (here refers to the increase of the maximum value of external stimulus current $I$ required to maintain the stability of the equilibrium point, i.e., $I_{H}^{01}>I_{H}$ ). Moreover, compared with Fig.4, the system (1) only has a bistable region with one structure, that is, there is only the coexistence region of the quiescent state and the period-1 spiking, while the area of the quiescent state coexists with the period-2 bursting disappears. It indicates that the bursting activities of the model are inhibited, and it is different from the traditional view that positive feedback can enhance the bursting activities of neurons [66]. Meanwhile, the anti-interference ability of the system (1) is enhanced (here refers to the increase of the minimum limit of external stimulus current $I$ required to stimulate electrical activities in the bistable region, i.e., $I_{*}^{01}>I_{*}$ ), and the bistable range is reduced $\Delta I_{01}<\Delta I$ (where $\Delta I=I_{H}-I_{*}=0.0681, \Delta I_{0 i}=I_{H}^{0 i}-I_{*}^{0 i}$, $(i=1,2, \mathrm{~L}, 6)$, so $\left.\Delta I_{01}=0.0622\right)$. Similarly, the results in Figs. 8 (b) and (c) testify that the stability and anti-interference ability of the system (1) continue to improve with the gradual increase of parameter $g_{M}$ (i.e., $I_{H}^{03}>I_{H}^{02}>I_{H}^{01}$ and $I_{*}^{03}>I_{*}^{02}>I_{*}^{01}$ ), but the corresponding bistable range continues to shrink (i.e., $\Delta I_{03}<\Delta I_{02}<\Delta I_{01}$ ). The results testify that the positive feedback memristive autaptic current can improve the stability and anti-interference ability of the system (1) by inhibiting its bursting activities, thus reducing the bistable or hidden firing patterns range of the model.

In particular, negative feedback memristive autaptic currents $I_{M}$ play an important role in promoting bursting activities in the system (1). When $g_{M}=-0.1$, Fig. 8(d) displays that the Hopf bifurcation behavior of the system (1) occurs at parameter $I_{H}^{04}=1.062163$. It is observed that the firing interval of period-2 bursting of the model increases significantly. Intuitively, compared with Fig. 4, although the system (1) also has the bistable region of two structures, the area of the quiescent state and period-1 spiking coexist is significantly reduced. Meanwhile, the stability and antiinterference ability of the system (1) are weakened (i.e., $I_{H}^{04}<I_{H}$ and $I_{*}^{04}<I_{*}$ ), while the bistable range is increased (i.e., $\Delta I_{04}>\Delta I$ ). Importantly, as the negative feedback gain $g_{M}$ continues to enhance, the unusual phenomenon of the inhibitory effect promoting rather than decreasing the bursting activities of neurons becomes more intuitive. When $g_{M}=-0.2$, Fig. 8(e) exhibits that the Hopf bifurcation behavior occurs of the model at parameter $I_{H}^{05}=0.918902$. It is observed that the coexisting state of the quiescent state and period-3 bursting appears, and the coexisting region of the quiescent state and period-1 spiking disappears. Meanwhile, the stability and anti-interference capability of the model continue to weaken (i.e., $I_{H}^{05}<I_{H}^{04}$ and $I_{*}^{05}<I_{*}^{04}$ ), whereas the bistable range continues to expand (i.e., $\Delta I_{05}>\Delta I_{04}$ ). When $g_{M}=-0.3$, Fig. 8 (d) shows that the system (1) has three types of bistable regions that are composed of the coexistence of the quiescent state and the bursting patterns of period-2, 3, and 4, respectively. It can discover the stability and antiinterference ability of the system (1) are further weakened (i.e., $I_{H}^{06}<I_{H}^{05}$ and $I_{*}^{06}<I_{*}^{05}$ ), and the bistable range is further expanded (i.e., $\Delta I_{06}>\Delta I_{05}$ ). It is worth mentioning that this is the first case of neuronal models able to have three types of bistable structures simultaneously to our knowledge. These results suggest that negative feedback memristive autapse not only weakens the stability and anti-interference ability of the model but also promotes its bursting activities. 


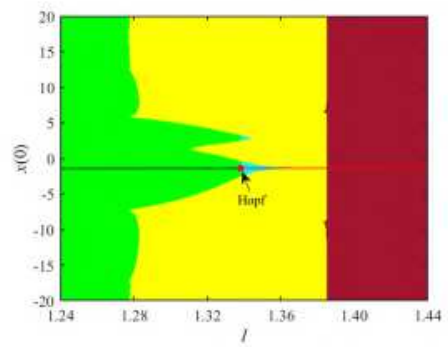

(a)

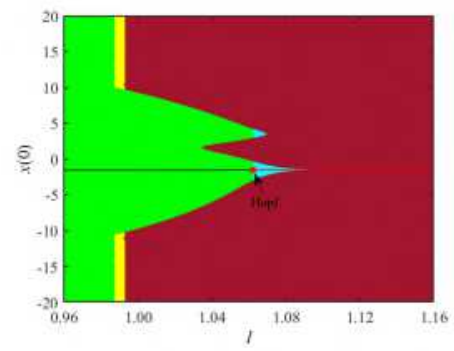

(d)

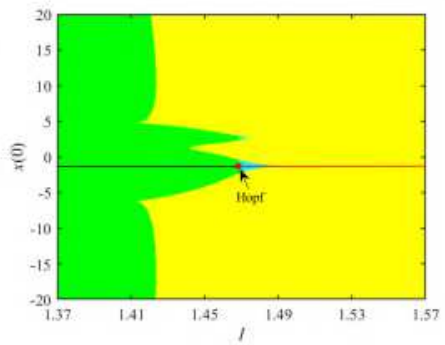

(b)

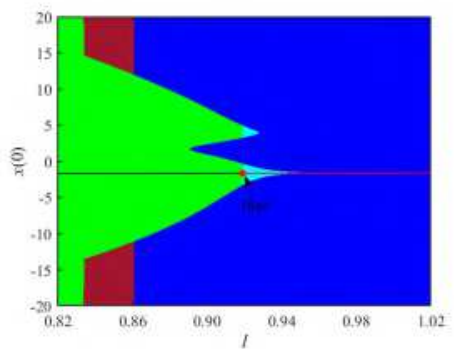

(e)

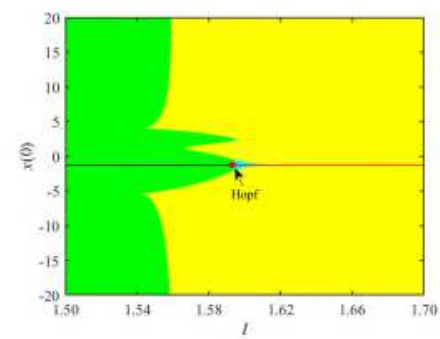

(c)

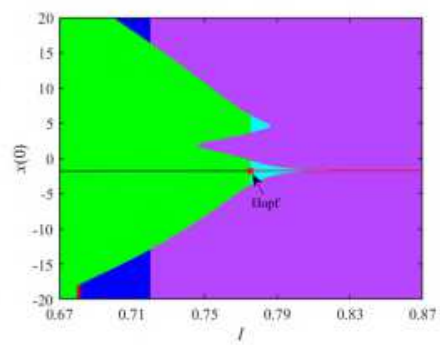

(f)

Fig 8 Global basins of attraction of the system (1) under memristive autaptic currents $I_{M}$ when $V_{e}=0$. The green area represents the quiescent state; The yellow area shows the period-1 spiking; The light blue area describes the subthreshold oscillations with increasing amplitude; The red area represents the period-2 bursting; The blue area indicates the period- 3 bursting; The purple area represents the period- 4 bursting; The black curve represents the stable equilibrium points; The red curve represents the unstable equilibrium points; The red dots are subcritical Hopf bifurcation points. (a) $g_{M}=0.1, I_{H}^{01}=1.338146, I_{*}^{01}=1.276$; (b) $g_{M}=0.2, I_{H}^{02}=1.468545, I_{*}^{02}=1.414$; (c) $g_{M}=0.3$, $I_{H}^{03}=1.593224, I_{*}^{03}=1.543$; (d) $g_{M}=-0.1, I_{H}^{04}=1.062163, I_{*}^{04}=0.9869$; (e) $g_{M}=-0.2, I_{H}^{05}=0.918902, I_{*}^{05}=0.8332$; (f) $g_{M}=-0.3, I_{H}^{06}=0.775221, I_{*}^{06}=0.6796$.

It should be recalled that Fig. 1(c) confirms that parameter $V_{e}$ can alter the characteristic curves of memristive autaptic current $I_{M}$ and membrane voltage $x$. Therefore, it is necessary to further analyze the regulation mechanism of parameter $V_{e}$ to memristive autaptic current $I_{M}$. For simplicity, let $V_{e}=-3.3$, Figs. 9(a)-(f) exhibit the global attraction domain of system (1) evolution results under the action of different types and intensities of memristive autaptic current $I_{M}$. When $g_{M}=0.1$, Fig. 9(a) shows that the subcritical Hopf bifurcation of system (1) occurs at parameter $I_{H}^{-1}=1.282005$. Compared with Fig. 4, it is observed that although system (1) also has the bistable region of two structures, the coexisting area of the quiescent state and period-1 spiking is significantly reduced. Accordingly, the coexisting region of the quiescent state and period-2 bursting is expanded. Moreover, the stability and anti-interference capability of the system are significantly enhanced (i.e., $I_{H}^{-1}>I_{H}$ and $I_{*}^{-1}>I_{*}$ ), and its bistable range is reduced (i.e., $\Delta I_{-1}<\Delta I$ ), (where $\Delta I_{-i}=I_{H}^{-i}-I_{*}^{-i},(i=1,2, \mathrm{~L}, 6)$, so $\left.\Delta I_{-1}=0.0660\right)$. Similarly, when $g_{M}=0.2$, the global basin of attraction of system (1) is shown in Fig. 9(b). It can be found that the Subcritical Hopf bifurcation behavior of the model occurs at parameter $I_{H}^{-2}=1.382841$, and a new bistable region consisting of the quiescent state and period-3 bursting has emerged. Noted that the region where the original quiescent state coexisted with the period-1 spiking disappeared, and the bistable region was further reduced (i.e., $\Delta I_{-2}<\Delta I_{-1}$ ). For $g_{M}=0.3$, Fig. 9(c) shows that system (1) has three types of bistable 
structures that the coexistence of the quiescent state and the bursting modes of period-2, 3, and 4 . It can discover the positive feedback memristive autapse can improve the stability and antiinterference ability of the model (i.e., $I_{H}^{-3}>I_{H}^{-2}>I_{H}^{-1}>I_{H}$ and $I_{*}^{-3}>I_{*}^{-2}>I_{*}^{-1}>I_{*}$ ). Moreover, the bistable involves a higher period of hidden firing activities, which is consistent with the traditional view that positive feedback stimulation can promote the excitability of neurons [66]. Nevertheless, the corresponding bistable range continue to be reduced (i.e., $\Delta I_{-3}<\Delta I_{-2}<\Delta I_{-1}<\Delta I$ ).

Conversely, the negative feedback memristive autaptic current $I_{M}$ can effectively inhibit the bursting activities of the system (1). When $g_{M}=-0.1$, Fig. 9(d) exhibits that the Hopf bifurcation behavior occurs in the system (1) at parameter $I_{H}^{-4}=1.147641<I_{H}$, and there is only a bistable structure composed of the quiescent state and period-1 spiking. Noted that the corresponding bistable range becomes larger (i.e., $\Delta I_{-4}>\Delta I$ ). Similarly, when $g_{M}=-0.2$, Fig. 9(e) shows that the stability and anti-interference ability of the system (1) continues to weaken (i.e., $I_{H}^{-5}<I_{H}^{-4}$ and $I_{*}^{-5}<I_{*}^{-4}$ ), and the bistable range is significantly expanded(i.e., $\Delta I_{-5}>\Delta I_{-4}$ ). Unexpectedly, when $g_{M}=-0.3$, the results in Fig. 9(f) testify that the stability and anti-interference capability of the system (1) are enhanced (i.e., $I_{H}^{-6}>I_{H}^{-5}$ and $I_{*}^{-6}>I_{*}^{-5}$ ). Note that this is different from the traditional view that negative feedback can inhibit the firing activities of neurons. Meanwhile, the bistable range will continue to increase (i.e., $\Delta I_{-6}>\Delta I_{-5}$ ). These results greatly enrich the researches on bistable types of neurons and provide a useful reference for understanding the mechanism of memristive autapse on neurons. Moreover, it indicates that parameters $g_{M}$ and $V_{e}$ can change the mechanism of memristive autaptic current $I_{M}$ on the neuron, so as to produce a counterintuitive dynamic phenomenon under the appropriate combination of parameters. Accordingly, it is very important and necessary to further reveal the dynamic mechanism.

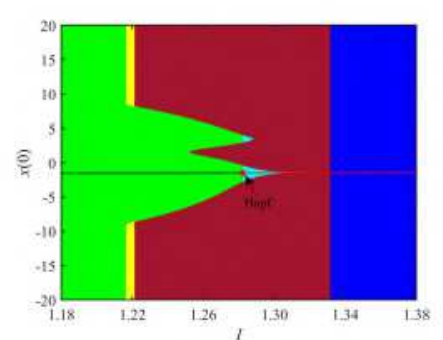

(a)

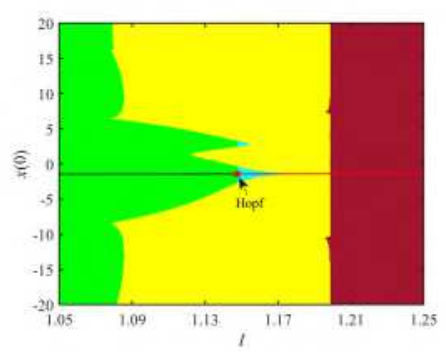

(d)

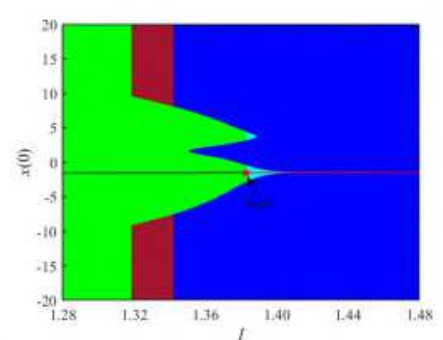

(b)

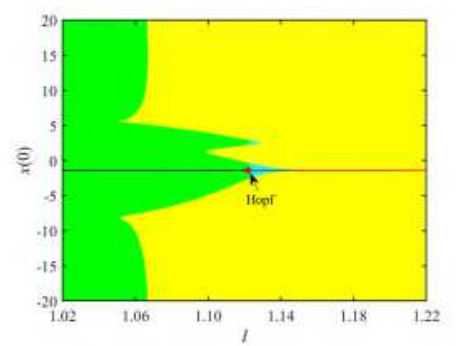

(e)

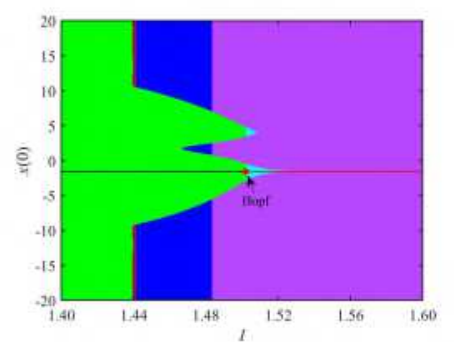

(c)

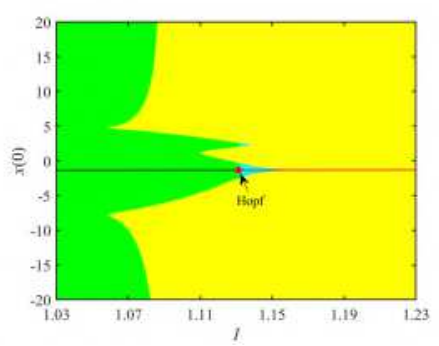

(f)

Fig. 9 The global basins of attraction of the system (1) under memristive autaptic currents $I_{M}$ when $V_{e}=-3.3$, where the meanings of each color region are consistent with those in Fig. 8. (a) $g_{M}=0.1, I_{H}^{-1}=1.282005, I_{*}^{-1}=1.216$; (b) $g_{M}=0.2, I_{H}^{-2}=1.382841, I_{*}^{-2}=1.318$; (c) $g_{M}=0.3, I_{H}^{-3}=1.501994, I_{*}^{-3}=1.439$; (d) $g_{M}=-0.1, I_{H}^{-4}=1.147641$, 
$I_{*}^{-4}=1.079$; (e) $g_{M}=-0.2, I_{H}^{-5}=1.122113, I_{*}^{-5}=1.052 ;$ (f) $g_{M}=-0.3, I_{H}^{-6}=1.131348, I_{*}^{-6}=1.058$.

\subsection{Counterintuitive phenomena and mechanisms}

To facilitate the measurement of the level of the excitatory or inhibitory effect of memristive autapse, it is necessary to calculate the mean value of memristive autaptic current $I_{A M}$ over continuous time $T$, which is expressed as

$$
I_{A M}=\frac{1}{T} \int_{0}^{T} I_{M} d t=\frac{1}{T} \int_{0}^{T} g_{M} w^{2}\left(V_{e}-x\right) d t
$$

where $T$ denotes the period of the corresponding periodic firing modes or $T=2000 / \mathrm{s}$ for chaotic firing activities in numerical calculation. It can be found that $I_{A M}>0$ represents the excitatory effect of memristive autaptic current $I_{M}$, whereas $I_{A M}<0$ denotes the inhibitory effect of memristive autaptic current $I_{M}$. Obviously, $I_{A M} \approx 0$ indicates that the regulatory effect of memristive autapse on neurons is almost negligible.

When $I=2.0$ and other parameters take reference values, Fig. 10(a)-(c) displays that the average memristive autaptic current, bifurcation diagram, and firing frequency of the system (1) on the $g_{M} \in[-0.4,0.4], V_{e} \in[-5.5,3.0]$ plane, respectively. Note that diverse color areas indicate different periods firing patterns in Fig. 10(b), and corresponding numbers are used to represent them in the color bar on the right side, i.e., the number 0 represents the quiescent state, the numbers 1 and 2 denote the period- 1 and period- 2 bursting states, respectively, particularly, the number 20 indicates the firing period greater than 19 or chaotic state. Obviously, the parameter $I_{A M} \approx 0$ in the yellow area of Fig. 10(a), corresponding firing mode is the period-3 bursting state (as shown in Fig. 10(b)), and its firing frequency is about 0.6 (as shown in Fig. 10(c)). Importantly, Figs. 10(a)(c) show that the bursting activities of the system (1) exhibit the counterintuitive dynamic phenomena when $I_{A M} \neq 0$. More intuitively, when $V_{e}=0.5$, the time response diagrams of the system (1) and memristive autaptic current $I_{M}$ under the action of parameter $g_{M}$ are depicted in Fig. 11(a)-(f), respectively. For $g_{M}=0.1$, the system (1) is in a period-3 bursting state, and its average memristive autaptic current $I_{A M}$ is greater than zero. Intriguingly, the firing period of the system (1) gradually decreases as parameter $g_{M}$ increases. For example, when $g_{M}=0.2$, the system (1) is in the period-2 bursting state, and the system (1) is in the period-1 spiking state for $g_{M}=0.3$. Conversely, when $g_{M}<0$, Figs. 11(d)-(f) testify that the number of spikes per burster of the system (1) increases rather than decreasing with the gradual enhancement of inhibited memristive autaptic current. It can discover the system (1) is in period-5, period-7, and period-9 bursting states when $g_{M}$ is set to $-0.1,-0.2$, and -0.3 , respectively. Consequently, the positive average memristive autaptic current can inhibit its excitability, while the negative average memristive autaptic current can increase its firing frequency or bursting activities. 


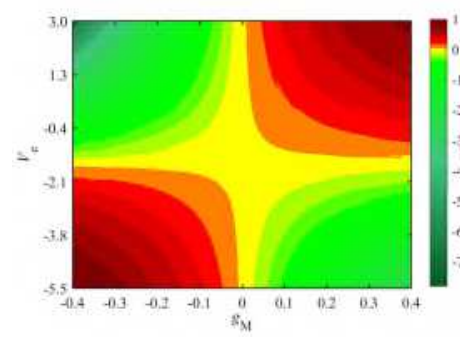

(a)

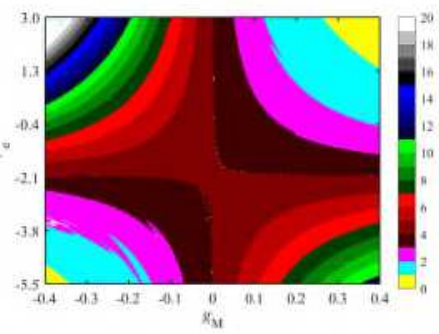

(b)

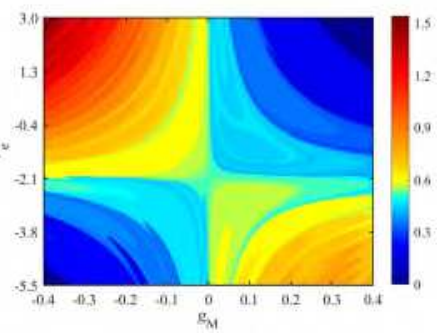

(c)

Fig. 10 The counterintuitive dynamic activities of the system (1) on the $g_{M} \in[-0.4,0.4], V_{e} \in[-5.5,3.0]$ plane when $I=2.0$. (a) Average memristive autaptic current $I_{A M}$; (b) Bifurcation diagram; (c) Firing frequency.

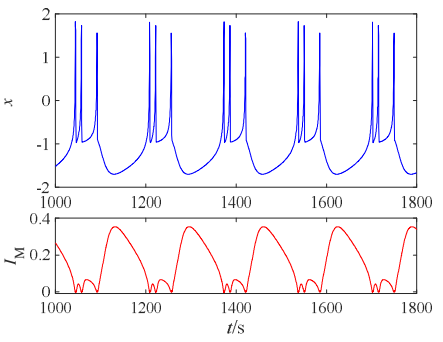

(a)

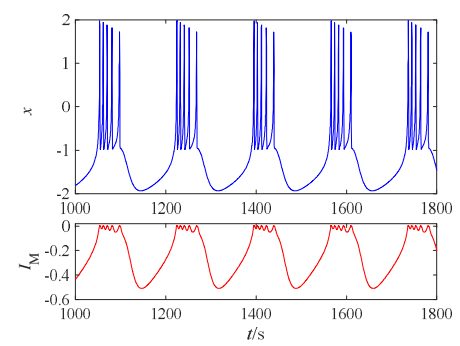

(d)

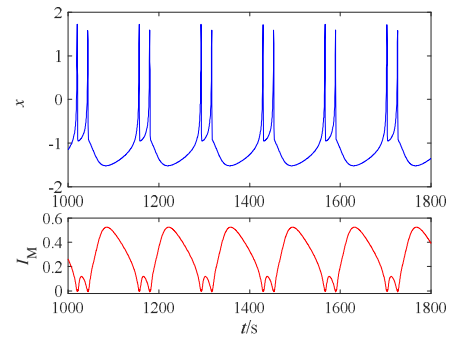

(b)

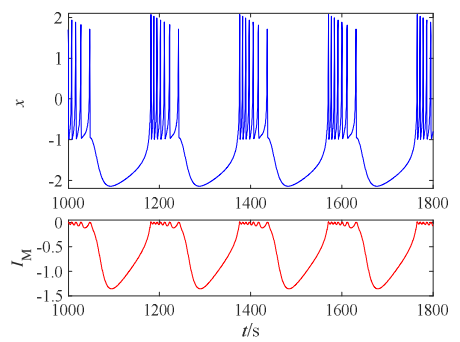

(e)

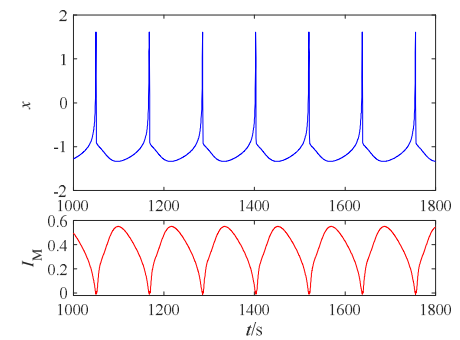

(c)

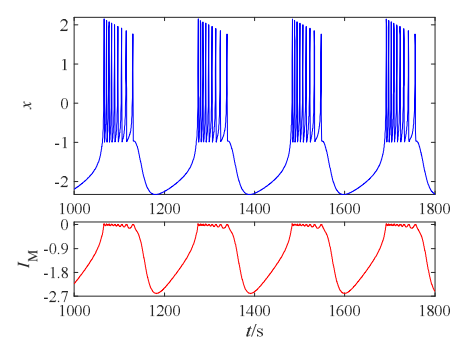

(f)

Fig. 11 The time response diagrams of the system (1) and memristive autaptic current $I_{M}$ at different feedback gain $g_{M}$ when $V_{e}=0.5$. (a) $g_{M}=0.1$; (b) $g_{M}=0.2$; (c) $g_{M}=0.3$; (d) $g_{M}=-0.1$; (e) $g_{M}=-0.2$; (f) $g_{M}=-0.3$.

To reveal the counterintuitive firing mechanism shown in Figs. 10 and 11, the fast-slow variable dissection and bifurcation analysis are feasible methods. Since system (1) has different time scales (i.e., $r=1$ ), this is also the internal cause of bursting patterns and MMOs. Accordingly, the fast subsystem contains four variables $x, y, E$, and $w$, which is the cause of generating repetitive firing pulses. The variable of the slow subsystem is $z$ that is responsible for regulating the alternating behavior between the quiescent state and the firing spikes. Figs. 12(a)-(d) show that the bifurcation diagrams of fast subsystem concerning slow variable $z$ and phase trajectories of the system (1) under different conditions of $g_{M}$, in which Fig. 12(b) and (d) is an enlargement of Fig. 12(a) and (c), respectively. It is observed that the fast subsystem has a Z-shaped curve composed of stable points (solid line), unstable points (dashed line), and corresponding bifurcation points [i.e., Hopf bifurcation point (red point), saddle node bifurcation point (black point SN), and homochromatic bifurcation point (purple point $\mathrm{SH}$ )]. Meanwhile, the stability of the equilibrium points of the fast subsystem change from stable a state to an unstable state, and a stable limit cycle is generated via the supercritical Hopf bifurcation when the slow variable $z \in[-12,4]$ gradually increases. Interestingly, Figs. 12(a) and (c) show that the maximum and minimum values of the limit cycle together form a $\mathrm{U}$-shaped solid line and terminate at the $\mathrm{SH}$ point of the fast subsystem. 
Moreover, the phase trajectories of these bursting modes start at the SN point and end at the $\mathrm{SH}$ point, so these firing patterns are usually referred to as "fold cycle/homoclinic" bursting patterns [4].

The counterintuitive phenomena caused by memristive autapse can be explained in terms of geometric dynamics. Notably, Figs. 12(a) and (b) exhibit that the SN point and SH point have a rightward shift in larger and smaller distances, respectively, with the gradual increase of parameter $g_{M}$, resulting in the gradual shortening of the distance between SN point and SH point of a burster. It is confirmed that the larger the positive feedback gain $g_{M}$ is, the smaller the number of spikes in the bursting states or firing frequency is. Conversely, Figs. 12(c) and (d) display that the SN point and SH point have a leftward move to larger and smaller distances, respectively, with the gradual enhancement of negative feedback gain $g_{M}$, leading to a gradual increase in the distance between SN point and SH point of a burster. It indicates that the number of spikes per burster or firing frequency will decrease gradually. These results will help to reveal the dynamic mechanism of bursting activities inhibited by the excitatory stimulus and promoted by the inhibitory stimulus.

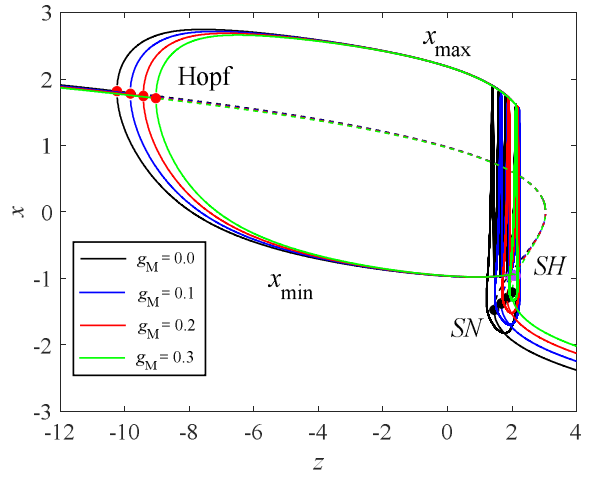

(a)

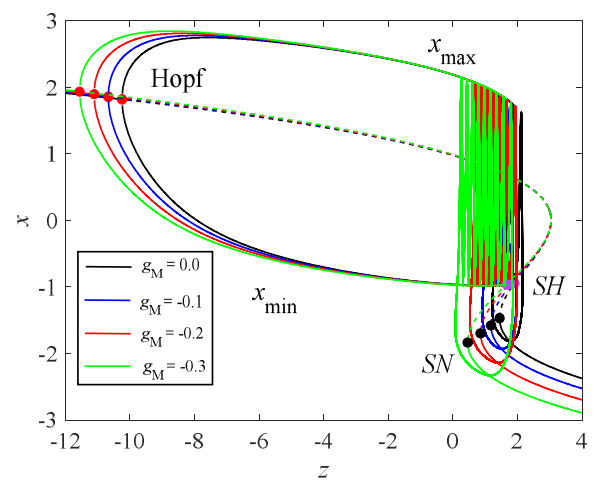

(c)

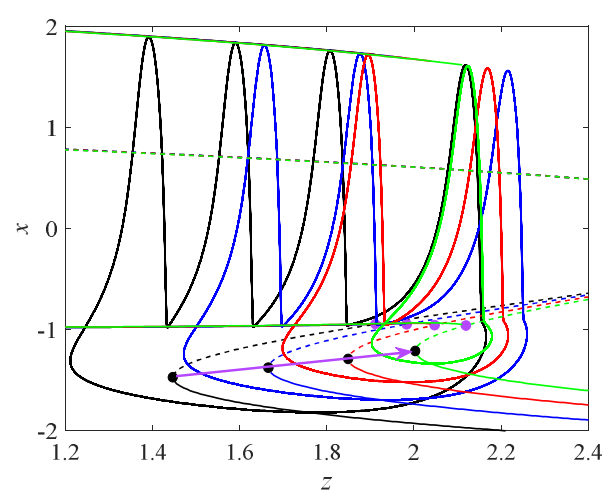

(b)

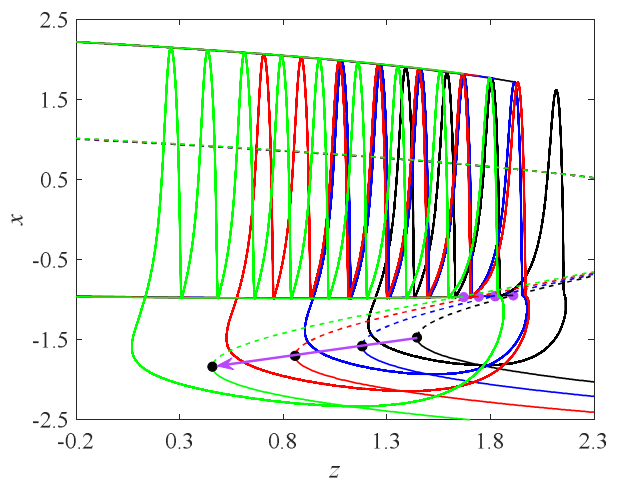

(d)

Fig. 12 Bifurcation behaviors of the fast subsystem and phase trajectories of the system (1) at different parameters $g_{M}$ when $V_{e}=0.5$. (a) The fast-slow dynamic analysis when $g_{M}=0.0,0.1,0.2$, and 0.3; (b) Enlargement of Fig. (a); (c) The fast-slow dynamic analysis when $g_{M}=0.0,-0.1,-0.2$, and -0.3; (d) Enlargement of Fig. (c).

\section{Self-organization, multistability, and synchronization}

\subsection{Comb-shaped structure}

Generally, the reference parameters of neuron models will be significantly disturbed by external stimulus and autaptic regulations, which may lead to changes in the electrical activities and even generate multistability or initial value sensitivity. Therefore, it is very important and necessary 
to explore the firing features of neurons in multi-parameter spaces. Figs. 13(a)-(f) exhibit the dynamic response diagram of the system (1) with self-similar characteristics on different twoparameter planes. Intuitively, the bifurcation diagram on the $I \in[2.5,3.6], r \in[0.001,0.021]$ plane is shown in Fig. 13(a). It can discover each color region with tongue-shaped (i.e., from light blue to light gray) is regularly distributed, accordingly, system (1) generates bursting activities from period2 to period-19, and the corresponding color regions become narrower with the increase of the number of spikes in a burster. Interestingly, these tongue-shaped regions enter chaotic or higher period firing modes via period-doubling bifurcation. However, due to the limitation of calculation accuracy, we cannot distinguish the firing type of system (1) in the white area. Accordingly, it is necessary to calculate the largest Lyapunov exponent diagram, as shown in Fig. 13(b), where the yellow region represents periodic firing modes because its corresponding largest Lyapunov exponent is very close to zero. Furthermore, the largest Lyapunov exponent is greater than zero in the red region with comb-shaped, which indicates the generation of chaotic firing activities. Meanwhile, this shows that Fig. 13(a) and (b) have good consistency and complementarity and confirms the system (1) has a classical comb-shaped chaotic structure.

To reveal the more detailed bifurcation features of the system (1), the bifurcation diagram and the largest Lyapunov exponent diagram along the direction shown by the purple line in Fig. 12 are shown in Fig. 14(a) and (b), respectively. It can be seen that as the parameter $I$ increases from 3.1 to 3.4, the period-adding bifurcation with chaos is organized as period-4 bursting $\rightarrow$ the chaotic state is entered via period-doubling bifurcation $\rightarrow$ the chaotic region is terminated via tangent bifurcation and generates period-5 bursting $\rightarrow$ the chaotic state is reentered via period-doubling bifurcation $\rightarrow \ldots \rightarrow$ period-19 bursting. Note that the corresponding periodic window becomes smaller with the increase of the bursting period in the bifurcation process, which conforms to the self-organization regulation of periodic regions in Fig. 13(a). Interestingly, the inter-spike interval of the firing series is rapidly shortened at parameter $I=3.376$, and this is also called the phenomenon of Shrinkage. Finally, the system (1) enters the period-1 spiking mode from the chaotic state via inverse period-doubling bifurcation. Moreover, compared with the onedimensional bifurcation diagram, Fig. 13(a) can show richer and more comprehensive dynamics details.

Actually, there are many parameters have a decisive effect on the firing activities of the model, including $I, g_{M}$, and $k_{0}$, etc. A natural question is to ask, what are the rules of regulation between these parameters to the firing activities of neurons? Therefore, Figs. 13(c)-(f) expand the bifurcation diagrams of the system (1) on these key parameter planes. It is observed that the combshaped fractal structure still exists in any combination of parameter spaces. Consequently, it can be predicted that the comb-shaped chaotic structure is the common feature of the system (1) in twodimensional parameter spaces.

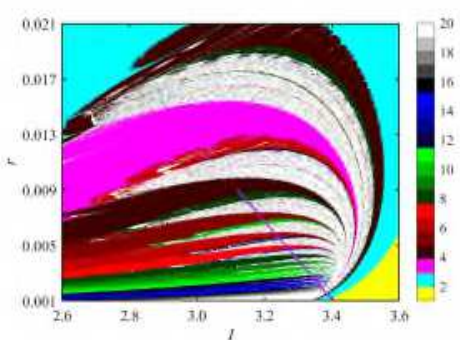

(a)

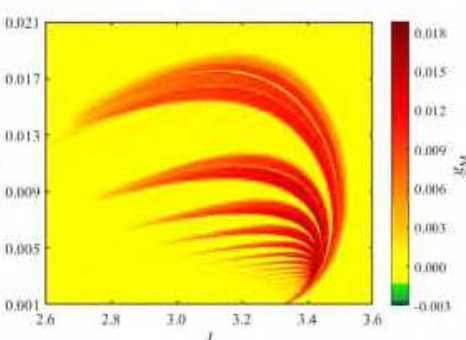

(b)

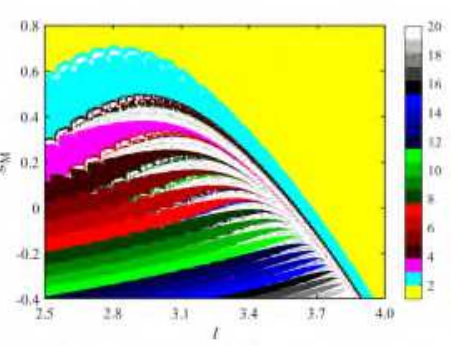

(c) 


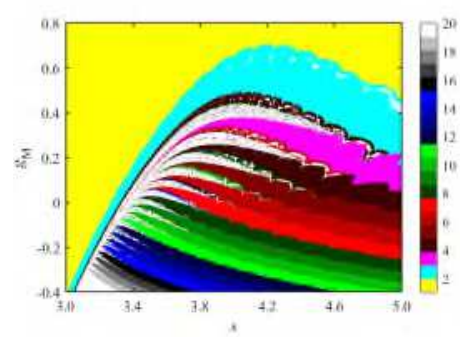

(d)

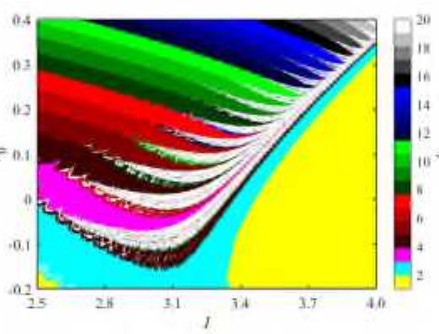

(e)

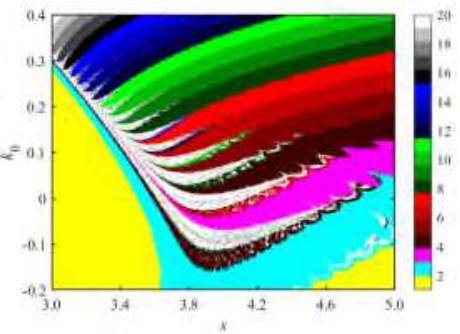

(f)

Fig. 13 Self-similar fractal features of the system (1) on different parameter planes. (a) Bifurcation diagram of $I \in[2.5,4.0], r \in[0.001,0.021]$; (b) The largest Lyapunov exponent diagram of Fig. (a); (c) Bifurcation diagram of $I \in[2.5,4.0], g_{M} \in[-0.4,0.8]$; (d) Bifurcation diagram of $s \in[3.0,5.0], g_{M} \in[-0.4,0.8]$; (e) Bifurcation diagram of $I \in[2.5,4.0], k_{0} \in[-0.2,0.4]$; (f) Bifurcation diagram of $s \in[3.0,5.0], k_{0} \in[-0.2,0.4]$.

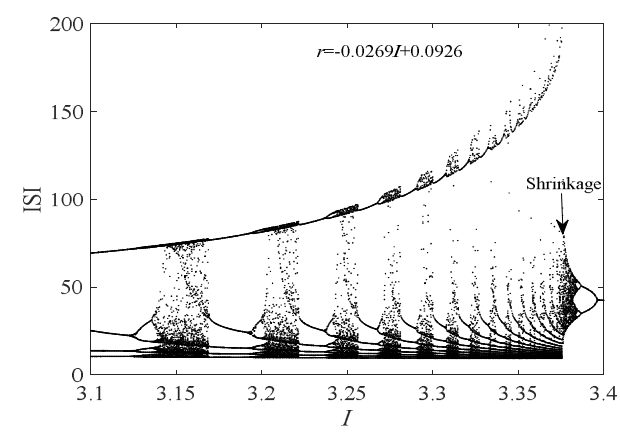

(a)

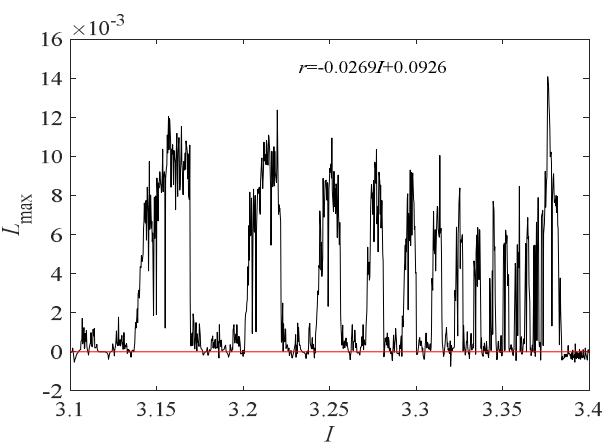

(b)

Fig. 14 The inter-spike interval (ISI) bifurcation diagram and largest Lyapunov exponent diagram of the system (1) along the direction shown by purple line $(r=-0.0269 I+0.0926, I \in[3.1,3.4])$ in Fig. 13(a).

\subsection{Locating coexisting attractors based on Hamiltonian energy}

Indeed, the dynamic systems produce various oscillating patterns that depend largely on the input and output of energy. Accordingly, the fluctuation of system energy can well reflect its oscillation states, that is, if the energy of the system shows periodic fluctuations, it indicates that the system is in periodic oscillations, while irregular energy injection and output will lead to chaos. Note that the period of energy oscillations does not necessarily coincide with the period of systems, especially systems with multiple time scales. Meanwhile, this section focuses on determining the firing modes of the model by analyzing the oscillation behaviors of the energy function. According to Helmholtz theorem [67], the system (1) can be expressed as the form of vector fields by

$$
X^{\&}=\left[\begin{array}{l}
x \\
\& \\
\& \\
\phi^{\&} \\
\&
\end{array}\right]=f(X)=\left[\begin{array}{c}
y-z-w \\
-d x^{2}+k_{0} E \\
r s x \\
k_{1} y \\
k_{3} x
\end{array}\right]+\left[\begin{array}{c}
b x^{2}-a x^{3}+w+I+I_{M} \\
c-y \\
-r s \chi_{0}-r z \\
-k_{2} E+E_{e x t} \\
-k_{4} w
\end{array}\right]=f_{c}(X)+f_{d}(X)
$$

where $f_{c}(X)$ represents the vortex field. $f_{d}(X)$ denotes the gradient field, and it can track and constrain the trajectories of the model. Furthermore, the Hamiltonian energy function $H(x, y, z, E, w)$ of the system (1) satisfies the relation as

$$
\nabla H^{T} f_{c}(X)=0, \text { and } \nabla H^{T} f_{d}(X)=d H / d t=I \& \text {. }
$$


For $\nabla H^{T} f_{c}(x)=0$, the equation can be obtained as

$$
(y-z-w) \frac{\partial H}{\partial x}+\left(-d x^{2}+k_{0} E\right) \frac{\partial H}{\partial y}+r s x \frac{\partial H}{\partial z}+k_{1} y \frac{\partial H}{\partial E}+k_{3} x \frac{\partial H}{\partial w}=0
$$

It can be easily obtained by calculating the Eq. (10)

$$
H(x, y, z, E, w)=\frac{1}{3} d x^{3}+\frac{1}{2}\left(r s+k_{0} k_{1}+k_{3}\right) x^{2}+\frac{1}{2}(y-z-w)^{2}+\frac{k_{0} k_{1}}{2 r s} z^{2}+\frac{k_{0} k_{1}}{2 k_{3}} w^{2}-k_{0} x E
$$

To ensure the uniqueness of the Hamilton energy, it is necessary to verify the relation $I^{\&}=\nabla H^{T} f_{d}(x)$, accordingly, the derivative of Hamilton energy $H$ can be obtained from

$$
\begin{aligned}
& I \&=\left(d x^{2}+\left(r s+k_{0} k_{1}+k_{3}\right) x-k_{0} E\right) \text { \& }(y-z-w) \&+\left(\frac{k_{0} k_{1}}{r s} z-(y-z-w)\right) \& k_{0} x \&+\left(\frac{k_{0} k_{1}}{k_{3}} w-(y-z-w)\right) L^{\&} \\
& =\left(d x^{2}+\left(r s+k_{0} k_{1}+k_{3}\right) x-k_{0} E\right)\left(y-a x^{3}+b x^{2}-z+I+I_{M}-w+w\right)+(y-z-w)\left(c-d x^{2}-y+k_{0} E\right) \\
& +\left(\frac{k_{0} k_{1}}{r s} z-(y-z-w)\right)\left(r\left(s\left(x-\chi_{0}\right)-z\right)\right)-k_{0} x\left(k_{1} y-k_{2} E+E_{e x t}\right)+\left(\frac{k_{0} k_{1}}{k_{3}} w-(y-z-w)\right)\left(k_{3} x-k_{4} w\right) \\
& =\left(d x^{2}+\left(r s+k_{0} k_{1}+k_{3}\right) x-k_{0} E\right)(y-z-w)+\left(d x^{2}+\left(r s+k_{0} k_{1}+k_{3}\right) x-k_{0} E\right)\left(b x^{2}-a x^{3}+I+I_{M}+w\right) \\
& \left.+(y-z-w)\left(-d x+k_{0} E\right)+(y-z-w)(c-y)+\left(\frac{k_{0} k_{1}}{r s} z-(y-z-w)\right)(r s x)+\left(\frac{k_{0} k_{1}}{r s} z-(y-z-w)\right)\left(-r s \chi_{0}-r z\right)\right) \\
& -k_{0} x\left(k_{1} y\right)-k_{0} x\left(-k_{2} E+E_{e x t}\right)+\left(\frac{k_{0} k_{1}}{k_{3}} w-(y-z-w)\right) k_{3} x+\left(\frac{k_{0} k_{1}}{k_{3}} w-(y-z-w)\right)\left(-k_{4} w\right) \\
& +\left(d x^{2}+\left(r s+k_{0} k_{1}+k_{3}\right) x-k_{0} E\right)\left(b x^{2}-a x^{3}+I+I_{M}+w\right)+(y-z-w)(c-y) \\
& r s \\
& =\nabla H^{T} f_{d}(X)
\end{aligned}
$$

The above calculation shows that Eq. (11) is the Hamilton energy of the system (1). Considering the Hamilton energy is closely related to the oscillation states of the model. It is worth exploring whether the Hamiltonian energy is also sensitive to initial values in the region of multistability of the model. Accordingly, can we accurately predict the multistability of the system by analyzing the initial sensitivity of the Hamiltonian energy? For simplicity, let $I=1.3$ and the other parameters were set as reference values. Figs. 15(a), (c) and Figs. 15(b), (d) exhibit the bifurcation diagrams of the energy $H$ and the system (1) on the $A \in[0,1.4], k_{0} \in[0.02,0.24]$ plane, respectively. It should be emphasized that the initial values of these numerical results are $(0.1,0.1$, $0.1,0.1,0.1,0.1)$ in the process of numerical simulation, while the difference is the direction of their numerical continuation (the result of each iteration serves as the initial value of the next iteration). In other words, Figs. 15(a) and (b) were obtained by gradually increasing the parameters $A, k_{0}$, conversely, the numerical continuation of Figs. 15(c) and (d) were by gradually decreasing the value of the parameters $A, k_{0}$. These numerical results confirm that the period of the energy $H$ is not the same as the firing period of the system (1), but their oscillation type is consistent. More precisely, if energy $H$ is in periodic oscillations, then system (1) is also in periodic firing states, otherwise, it indicates that the system (1) is in chaotic firing when energy $H$ is chaotic oscillations.

Furthermore, considering that the system (1) is a non-autonomous continuous system, it can be inferred that which superimposed colors in Figs. 15(a)-(d) (i.e., the inconsistent regions in Fig. 15(a) and (c) or Fig. 15(b) and (d)) are caused by the multistability of the model. Importantly, it is observed that the superimposed regions of energy $H$ are the same as that of the system (1), indicating that it is entirely feasible to determine the initial value sensitivity of the model by locating the multistability regions of energy $H$. In detail, for $\left(A, k_{0}\right)=(0.3395,0.1038)$, Fig. 16(a1) shows 
that energy $H$ at the initial value $(1.2,-4.1,0.1,0.1,0.1)$ and $(1.2,4.1,0.1,0.1,0.1)$ is oscillating in the MMOs of period- $2^{1}$ and period- $1^{2}$, respectively. Noted that the period- $L^{S}$ MMOs means that the number of large-amplitude and small-amplitude is $L$ and $S$, respectively, within an oscillation period. Accordingly, the system (1) is in the period-1 and the period-2 bursting, respectively, and its time response, phase trajectory, and fractal basin of attraction on the $x(0)-y(0)$ plane are depicted in Fig. 16(b1), (c1), and 17(a), respectively. Similarly, for $\left(A, k_{0}\right)=$ $(0.6818,0.1496)$, the energy $H$ is in the oscillatory coexistence state of period- $2^{2}$ and period- $2^{3}$ MMOs, meanwhile, the system (1) is in the coexistence state of period-2 and period-3 bursting patterns, and its dynamic responses are shown in Figs. 16(a2) - (c2) and 17(b). When $\left(A, k_{0}\right)=$ $(0.8697,0.2206)$, the energy $H$ is in the oscillatory coexistence state of period- $2^{3}$ and period- $2^{4}$ MMOs, and the system (1) is in the coexistence state of period-3 and period-4 bursting patterns, correspondingly, the dynamic responses are depicted in Figs. 16(a3) - (c3) and 17(c). When $\left(A, k_{0}\right)$ $=(0.9702,0.2066)$, the energy $H$ is in the oscillatory coexistence state of period- $2^{4}$ and chaotic MMOs, accordingly, the system (1) is in the coexistence state of period-4 and chaotic bursting patterns, and the dynamic responses are shown in Figs. 16(a4) - (c4) and 17(d). Unexpectedly, these numerical results show that the small-amplitude number of the energy $H$ is consistent with the firing period of the system (1). Moreover, the energy is larger when the model is in subthreshold oscillations. The internal mechanism is that each firing activity of the model consumes energy, resulting in energy reduction. However, the electric activities enter the subthreshold oscillation state by absorbing energy, leading to the decrease of system stability, and thus produces the next firing activities alternately. It also reveals the internal mechanism of bursting patterns from the perspective of energy consumption and supply.

The initial value sensitivity of the system (1) is a feature for the range of the particular parameters rather than some isolated parameter points. Notably, when $A \in[0.9696,0.9708]$, $k_{0}=0.2066$, Fig. 17(e) displays the space-time response of the evolution of the basin of attraction. We can observe that parameter $A$ has a decisive influence on the sensitivity of the initial value of the model, that is, the region of the period-4 bursting state is smaller, and the corresponding chaotic firing state is larger with the increase of parameter $A$. This is due to the larger amplitude $A$ of external electric field can effectively induce chaotic firing activities. Similarly, Fig. 17(f) exhibits the space-time response of the evolution of the basin of attraction when $k_{0} \in[0.2060,0.2072]$, $A=0.9702$. It is observed that with the increase of induced electric field intensity $k_{0}$, the larger the area of period-4 bursting is, accordingly, the smaller the corresponding region of chaotic firing is. These results indicate that the parameter $k_{0}$ plays an important role in promoting the regular (periodic) firing activities of neurons.

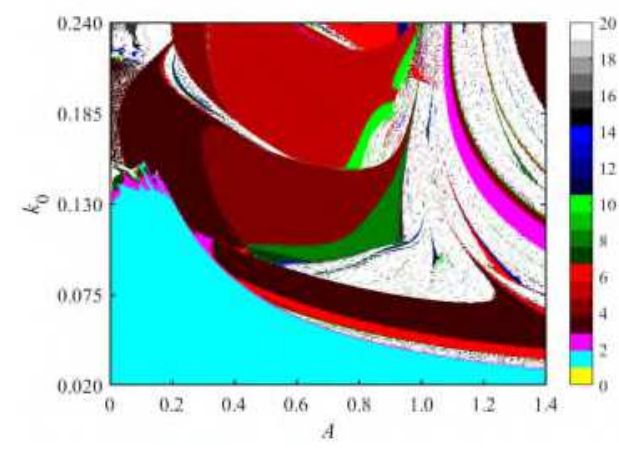

(a)

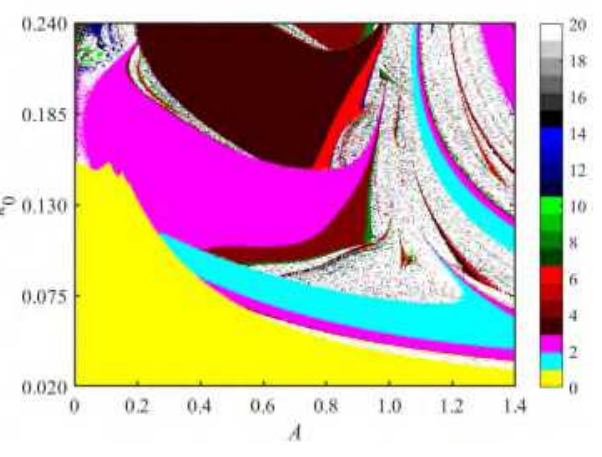

(b) 


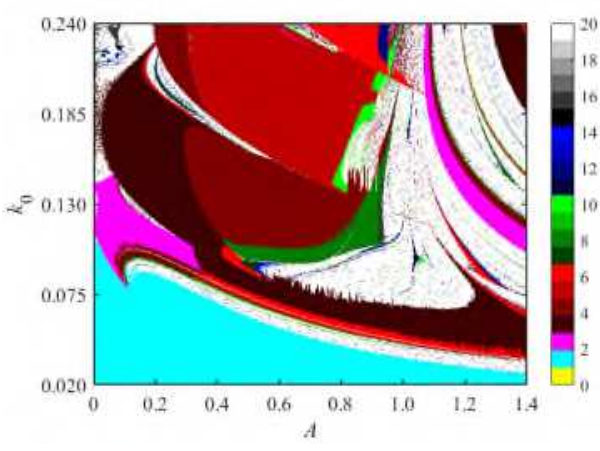

(c)

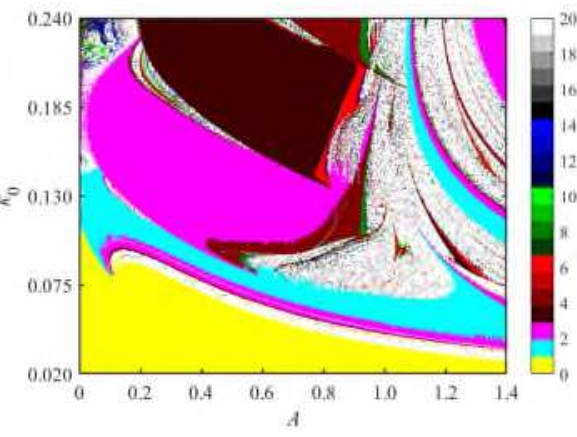

(d)

Fig. 15 Bifurcation diagrams of the system (1) and the Hamilton energy $H$ under different numerical continuation directions when $A \in[0,1.4], k_{0} \in[0.02,0.24]$. (a) Bifurcation diagram of energy $H$ and (b) bifurcation diagram of the system (1) is obtained by the numerical continuation of gradually increasing the parameters $A$ and $k_{0}$. (c) Bifurcation diagram of energy $H$ and (d) bifurcation diagram of the system (1) is obtained by the numerical continuation of gradually decreasing the parameters $A$ and $k_{0}$.

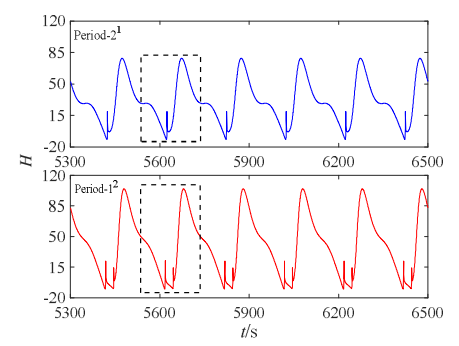

(a1)

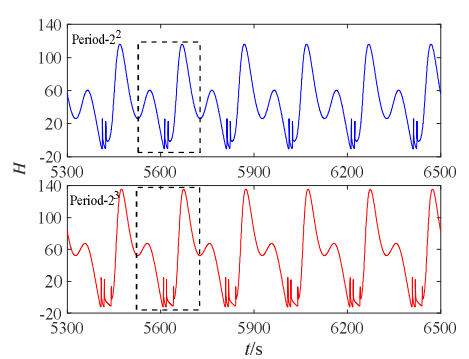

(a2)

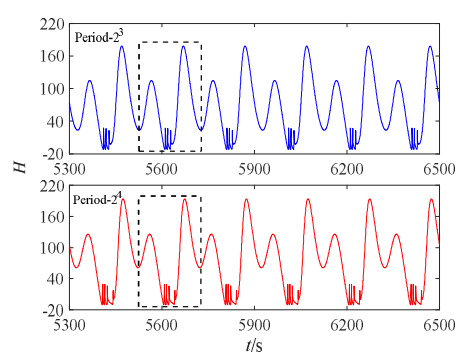

(a3)

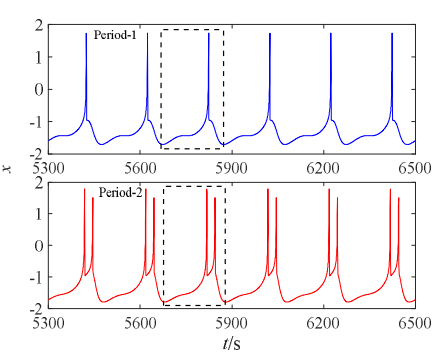

(b1)

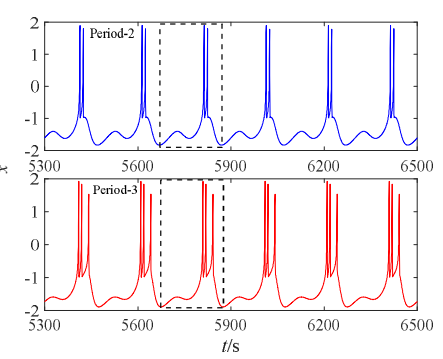

(b2)

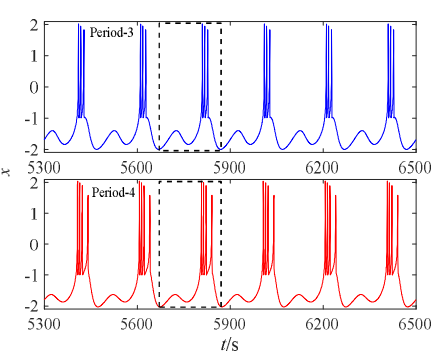

(b3)

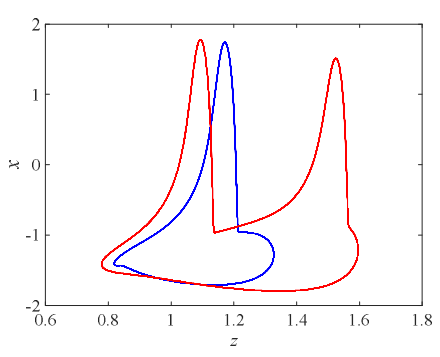

(c1)

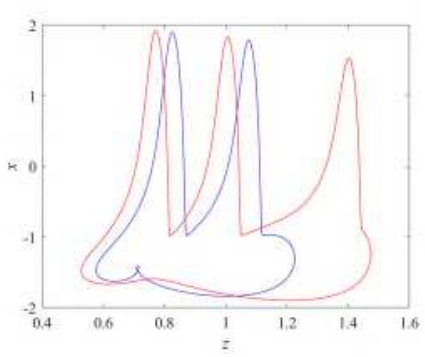

(c2)

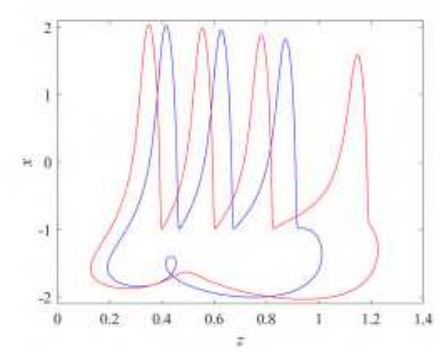

(c3) 


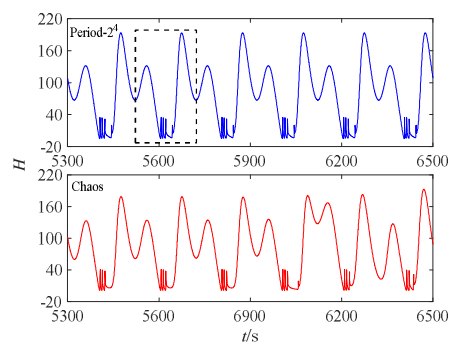

(a4)

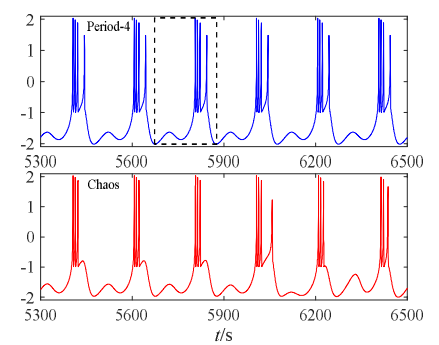

(b4)

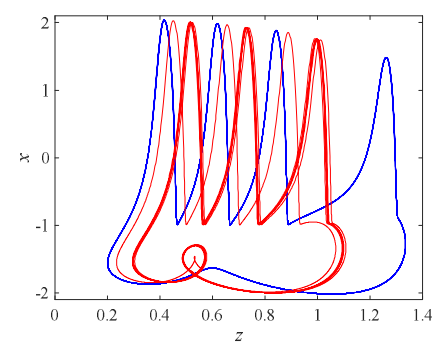

(c4)

Fig. 16 The dynamic responses of the system (1) and the Hamilton energy $H$ at different parameters $A$ and $k_{0}$. (a1), (a2), (a3), and (a4) Time response diagrams of energy $H$; (b1), (b2), (b3), and (b4) Time response diagrams of membrane voltage $x$; (c1), (c2), (c3), and (c4) Phase trajectory. (a1)-(c1) $\left(A, k_{0}\right)=(0.3395,0.1038)$; (a2)-(c2) $\left(A, k_{0}\right)=(0.6818,0.1496) ;(\mathrm{a} 3)-(\mathrm{c} 3)\left(A, k_{0}\right)=(0.8697,0.2206) ;(\mathrm{a} 4)-(\mathrm{c} 4)\left(A, k_{0}\right)=(0.9702,0.2066)$.

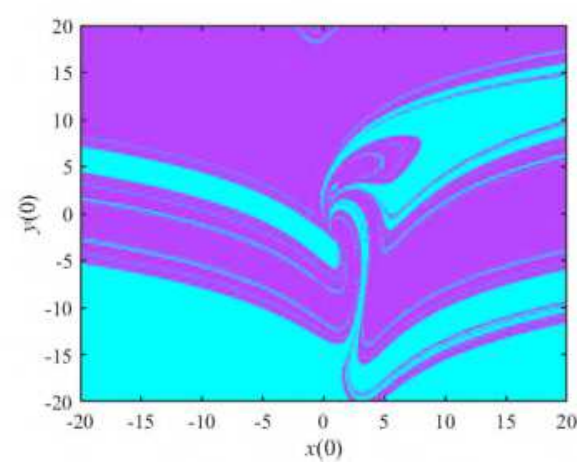

(a)

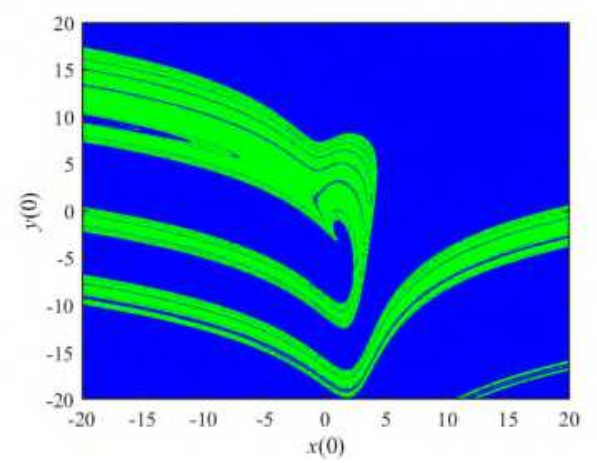

(c)

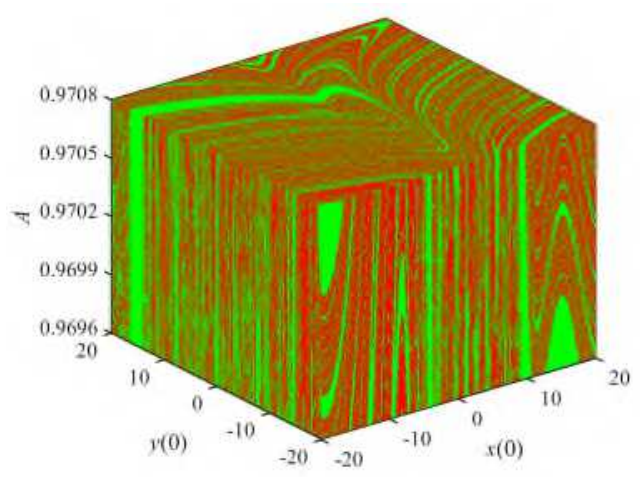

(e)

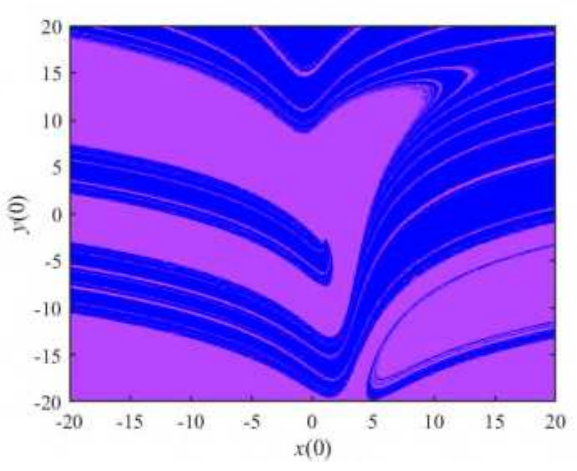

(b)

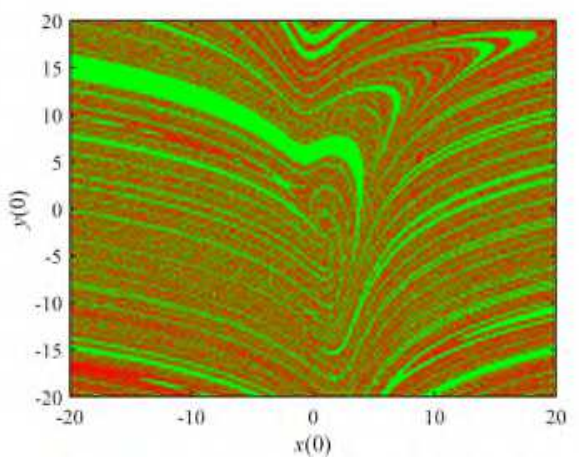

(d)

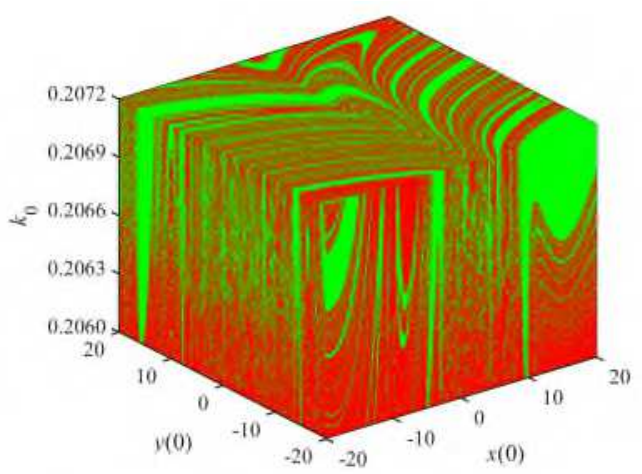

(f)

Fig. 17. Basins of attraction of the system (1) for to parameters $A$ and $k_{0}$ on the $x(0)-y(0)$ plane. The light blue area indicates the period-1 spiking. The purple area denotes the period-2 bursting; The blue area represents the 
period-3 bursting; The green area represents the period-4 bursting; The red area represents chaotic firing patterns. (a) $\left(A, k_{0}\right)=(0.3395,0.1038)$; (b) $\left(A, k_{0}\right)=(0.6818,0.1496)$; (c) $\left(A, k_{0}\right)=(0.8697,0.2206)$; (d) $\left(A, k_{0}\right)=(0.9702$, 0.2066), (e) $A \in[0.9696,0.9708], k_{0}=0.2066$; (f) $k_{0} \in[0.2060,0.2072], A=0.9702$. Note that Figs. (e) and (f) confirm that the chaotic regions will be larger with the increase of parameter $A$ or decrease of parameter $k_{0}$.

\subsection{Synchronization with unidirectional coupling involving energy}

Considering that the firing process of neurons is accompanied by energy injection and release, it is worth further investigating whether the synchronous control of the model can be achieved by unidirectional energy input. More importantly, synchronous behaviors of the unidirectional coupling system with mismatched parameters can be achieved by setting an intermittent Hamilton energy feedback controller. To verify that the intermittent controller involving energy $H$ is far more efficient and consumes less energy than the traditional electrical synaptic coupling controller for chaotic or synchronous control. It is necessary to first analyze the synchronous behaviors of the unidirectional electrical synapses coupling system, and the coupling model as

$$
\left\{\begin{array}{l}
\underset{i}{\&}=y_{i}-a x_{i}^{3}+b x_{i}^{2}-z_{i}+I+I_{M}+\Gamma_{i}, \\
\&=c-d x_{i}^{2}-y_{i}+k_{0} E_{i}, \\
\underset{t}{\&}=r\left[s\left(x_{i}-\chi_{0}\right)-z_{i}\right], \\
\&_{i}^{\&}=k_{1} y_{i}-k_{2} E_{i}+E_{e x t}, \\
\mathbb{\&}_{i}^{\&}=k_{3} x_{i}-k_{4} w_{i} .
\end{array}\right.
$$

where $\Gamma_{i}=-D\left(x_{i}-x_{j}\right)$ is a classical electrical synaptic coupling controller, whose purpose is to control from the membrane voltage $x_{i}$ of the slave system to the membrane voltage $x_{j}$ of the master system. To simplify the analysis, one master system and three slave systems are selected and denoted as case- $j$ and case- $i(j=0, i=1,2,3)$, respectively. Without loss of generality, the parameters and initial values of the master-slave system (12) are shown in Table 1. Clearly, Fig. 17(e) shows that the slave system Case- $i(i=1,2,3)$ are all sensitive to initial values and can be made to be in chaotic firing states by selecting appropriate initial values. Noted that the parameters of the slave system Case- 1 and the master system case- 0 are the same but the initial values are different, and the time response and phase trajectory are shown in red curves in Fig.16 (b4) and (c4), respectively. Moreover, the parameters and initial values of the slave systems Case-2/3 and the master system Case- 0 are inconsistent. To quantitatively characterize the complete synchronization behavior of the system (12), it is necessary to define the error function as

$$
e_{i}=\sqrt{\left(x_{i}-x_{j}\right)^{2}+\left(y_{i}-y_{j}\right)^{2}+\left(z_{i}-z_{j}\right)^{2}+\left(E_{i}-E_{j}\right)^{2}+\left(w_{i}-w_{j}\right)^{2}},(i=1,2,3, j=0) .
$$

Obviously, the system (12) realizes complete synchronization when the error function $e_{i}$ gradually approaches zero. Figs. 18(a)-(d) exhibit the states of phase synchronization and complete synchronization of the system (12) under different coupling strengths $D$. For $D=0$, the master system Case- 0 and the slave systems Case- $1 / 2 / 3$ respectively maintain independent discharge state, as shown in Fig. 18(a). Interestingly, when $D=0.6$, Fig. 18(b) confirms that the system (12) achieves phase synchronization but cannot attain complete synchronization. When $D=0.7$, Fig. 18(c) shows that complete synchronization of the slave system case- 1 is achieved (i.e., $e_{1}=0.01$ ), which also indicates that the sensitivity of the initial value of the slave system case- 1 is effectively controlled. However, the slave systems Case-2/3 cannot achieve complete synchronization, which 
is mainly because the parameter $A$ of the slave systems Case- $2 / 3$ is inconsistent with the parameter $A$ of the master system Case-0. Meanwhile, it requires a larger coupling strength $D$ to achieve the complete synchronous. The results in Fig. 18(d) testify that complete synchronization is also achieved for the slave systems case- $2 / 3$ only when $D=1.4$. Furthermore, considering that unidirectional electrical synaptic coupling inevitably produces energy loss in the process of realizing synchronization. Therefore, it is necessary to optimize the controller $\Gamma_{i}$ and reduce the coupling strength required to achieve complete synchronization of the system (12), thereby lowering the Joule heat and energy loss generated in the realization of synchronization [68].

Table 1 The firing patterns of the master system Case- $j(j=0)$ and the slave systems Case- $i(i=1,2,3)$ under the condition of different parameter values $A$ and initial values when $k_{0}=0.2066$.

\begin{tabular}{|c|c|c|c|}
\hline Case-j/i & Parameter values $A$ & Initial values & Firing patterns \\
\hline Case-0 & 0.9702 & $(1.2,2.1,0.1,0.1,0.1)$ & Period-4 \\
\hline Case-1 & 0.9702 & $(-1.2,2.1,0.1,0.1,0.1)$ & Chaos \\
\hline Case-2 & 0.9696 & $(-0.9,2.1,0.1,0.1,0.1)$ & Chaos \\
\hline Case-3 & 0.9708 & $(-0.7,2.1,0.1,0.1,0.1)$ & Chaos \\
\hline
\end{tabular}

Considering that energy injection, output, and exchange are accompanied in the synchronization process of the master-slave system so that the Hamilton energy of the master system Case- 0 is combined with the electrical synapse, and its expression is

$$
\bar{\Gamma}_{i}=-D\left(x_{i}-x_{j}\right)\left(\theta\left(H_{j}-H_{\min }\right)+\theta\left(H_{\max }-H_{j}\right)\right), H_{\min }<H_{\max }
$$

where $x_{j}$ and $H_{j}$ are the membrane voltage and Hamilton energy (abbreviated as $H$ ) of the master system case- $j(j=0)$, respectively. $\theta(\chi)$ is the Heavside function, and when $\chi>0$, $\theta(\chi)=1$, otherwise $\theta(\chi)=0 . H_{\min }$ and $H_{\max }$ are the adjustment parameters of the controller (14). More precisely, only when $H \in\left[H_{\text {min }}, H_{\text {max }}\right]$, the controller $\bar{\Gamma}_{i}$ can operate intermittently. Without loss of generality, parameters $H_{\min }=10$ and $H_{\max }=100$ are selected. When $D=0.1$, Fig. 19(a) confirms that complete synchronization of the system (12) cannot be realized. Meanwhile, it is observed that the system (12) has phase synchronization behavior when $t>3600 / \mathrm{s}$, and this is much less than the coupling strength $D=0.6$ required under the controller $\Gamma_{i}$ (i.e., Fig. 18(b)). Interestingly, when $D=0.25$, Fig. 19(b) displays that phase synchronization behavior of the slave system (12) is realized for $t>2000 / \mathrm{s}$. Furthermore, as parameter $D$ continues to increase to 0.4 , Fig. 19(c) shows that phase synchronization of the system (12) is achieved when $t>1000 / \mathrm{s}$ and complete synchronization of only the slave system Case-1 is attained. Importantly, when $D=0.7$, Fig. 19(d) shows that complete synchronization of the system (12) is achieved, and this is far more efficient than controller $\Gamma_{i}$ (i.e., Fig. 18(d)). These numerical results indicate that the control effect of the energy-containing intermittent controller $\bar{\Gamma}_{i}$ is much better than that of the conventional electrical synaptic coupling controller $\Gamma_{i}$, meanwhile, the joule heat and energy consumption generated by controller $\bar{\Gamma}_{i}$ are smaller. 


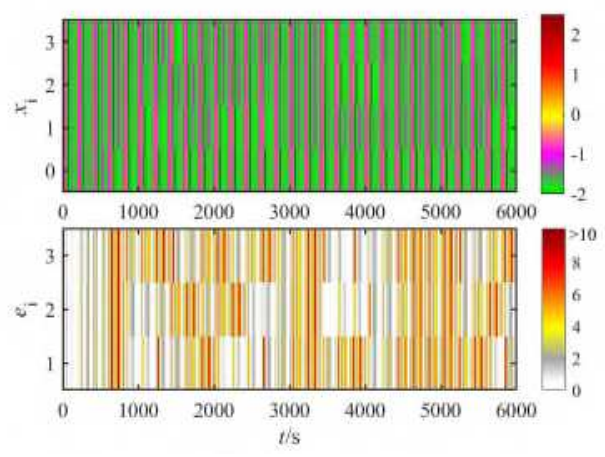

(a)
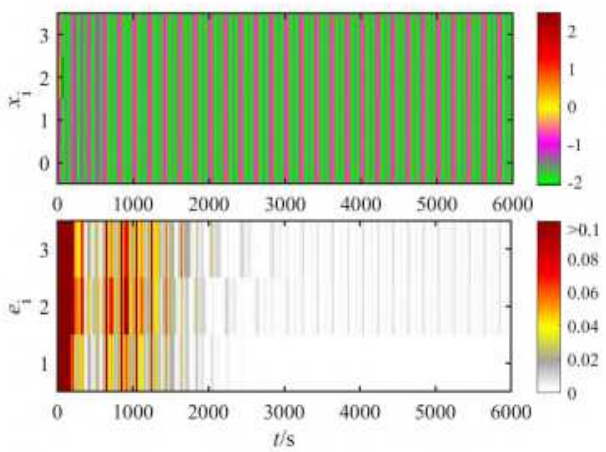

(c)

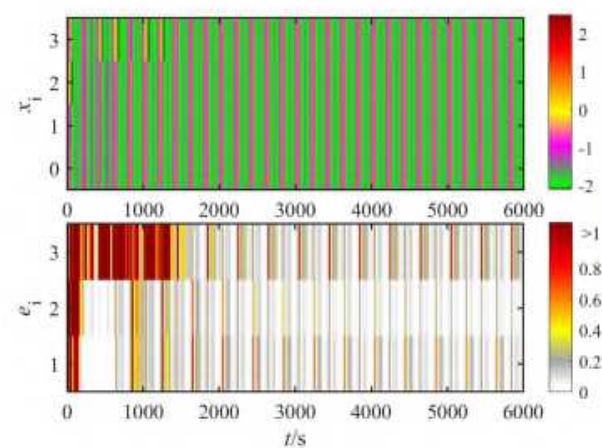

(b)

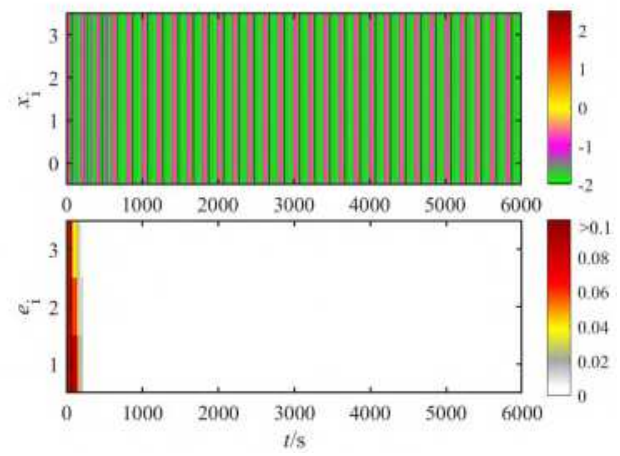

(d)

Fig. 18 Spatiotemporal response diagrams of phase synchronization and complete synchronization of the system (12) under the controller $\Gamma_{i}$. (a) $D=0$; (b) $D=0.6$; (c) $D=0.7$; (d) $D=1.4$.

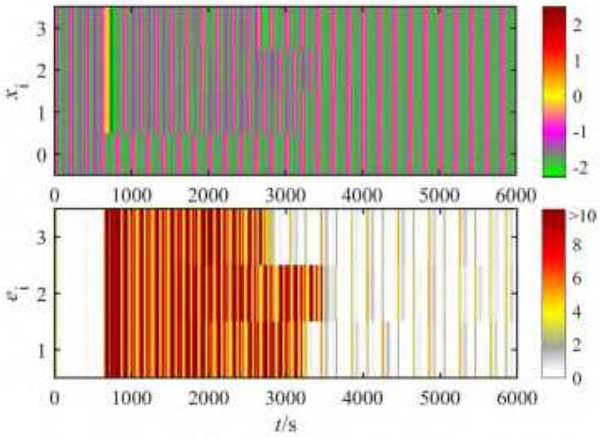

(a)

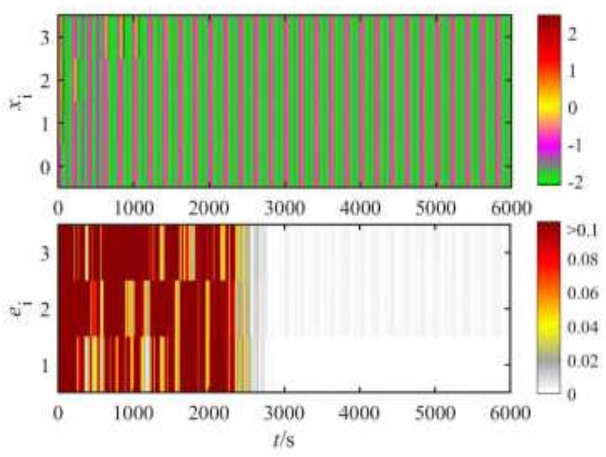

(c)

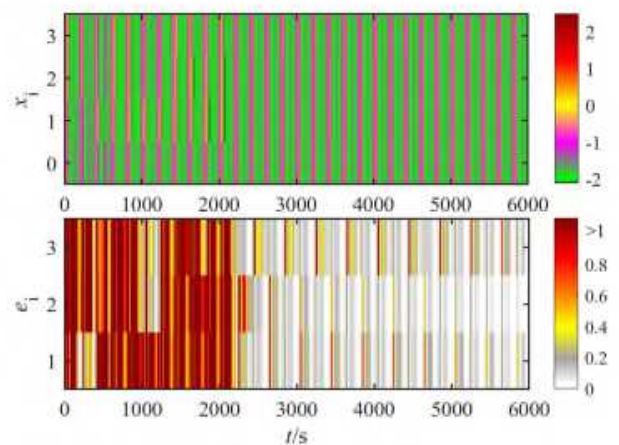

(b)

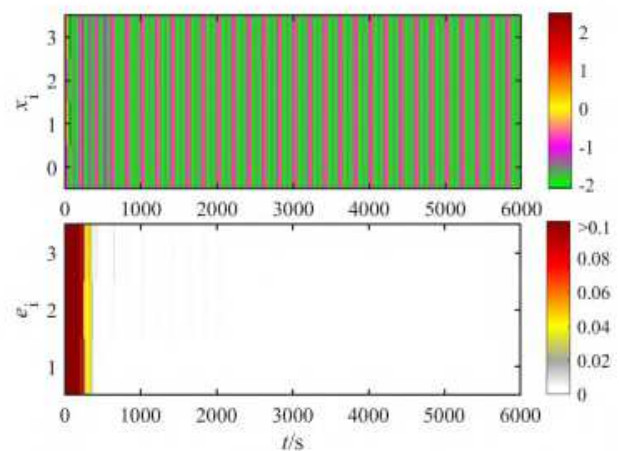

(d)

Fig. 19 Spatiotemporal response diagrams of phase synchronization and complete synchronization of the system (12) under the controller $\bar{\Gamma}_{i}$. (a) $D=0.1$; (b) $D=0.25$; (c) $D=0.4$; (d) $D=0.7$. 
To achieve the best control effect of the controller $\bar{\Gamma}_{i}$, especially for the synchronous control of the system with mismatched parameters (e.g., the slave systems case-2/3). It is necessary to study the optimal combination of parameters $H_{\min }$ and $H_{\max }$. Without loss of generality, we focus on analyzing the complete synchronization behavior of the slave system case-2, as shown in Figs. 20(a)-(f), where the green area represents the complete synchronized state, while the red area indicates the incomplete synchronized state. Figs. 20(a)-(c) confirm that parameter $H_{\max }$ has little influence on its synchronization state on the $D \in[0,1.5], H_{\min } \in[-20,60]$ plane, accordingly, the minimum coupling strength $D$ required to achieve complete synchronization of the slave system Case-2 mainly depends on the parameter $H_{\min }$. When $H_{\min } \in[-20,32]$, the slave system Case-2 achieves complete synchronization at $D=0.6493$, which is far less than the coupling strength $D=1.4$ required to achieves complete synchronization by the controller $\Gamma_{i}$. Moreover, Figs. 20(d)-(f) also confirm that the minimum coupling strength required to achieve complete synchronization of the slave system Case- 2 crucially relies on the value of parameter $H_{\min }$. Thus, it can be inferred that controller $\bar{\Gamma}_{i}$ has the optimal control effect on the slave system Case- 2 when $H_{\min } \in[-20,32], H_{\max } \in[61,201]$. Meanwhile, it should be emphasized that the optimal combination of parameters $H_{\min }$ and $H_{\max }$ can be obtained based on the above numerical method to achieve the optimal synchronous control effect for other slave systems with mismatched parameters.

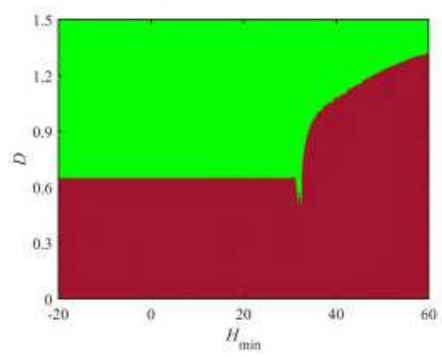

(a)

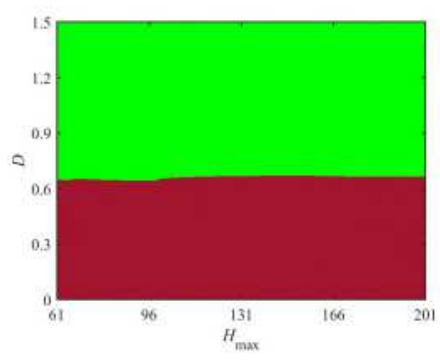

(d)

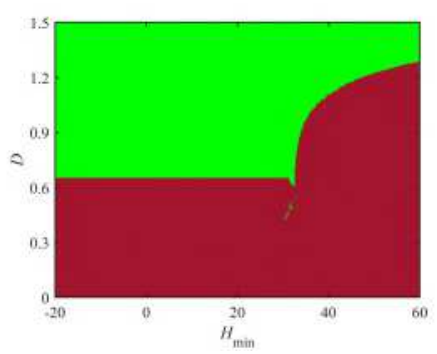

(b)

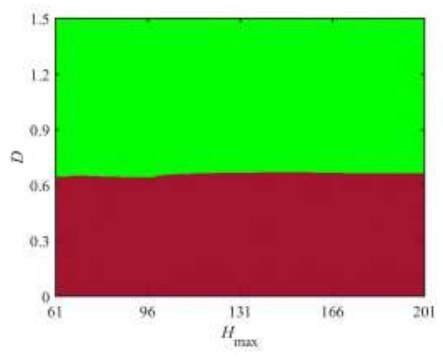

(e)

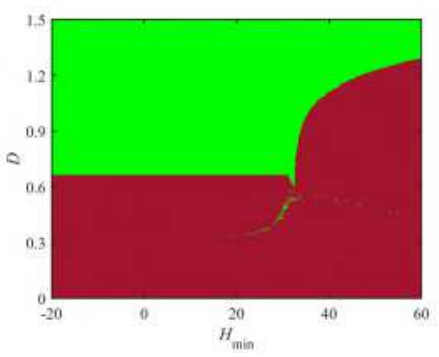

(c)

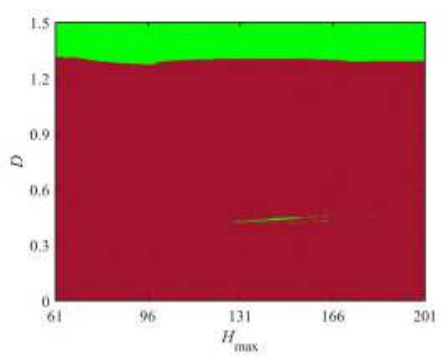

(f)

Fig. 20 The complete synchronization behavior of the system Case-2 under the regulation of parameters $D, H_{\min }$, and $H_{\max }$, in which the green area represents the complete synchronization and the red area indicates the incomplete synchronization. (a) $H_{\max }=61$; (b) $H_{\text {max }}=100$; (c) $H_{\max }=201$; (d) $H_{\text {min }}=-20$; (e) $H_{\min }=10$; (f) $H_{\text {min }}=60$.

\section{Conclusions}

Due to the fluctuation of extensive ions that will induce time-varying electromagnetic fields in the process of neural information transmission, a reliable neuron model should not ignore the combined modulation effect of electric field and magnetic field on electrical activities. Considering the characteristics of memristive autapse involving magnetic coupling, including voltage controlled, 
memory, and plasticity, it can well describe the feedback regulation of induction current on its membrane potential. Accordingly, the memristive autapse and electric field variables are introduced to construct a 5-D HR neuron model. It is worth mentioning that the model not only has a simple mathematical structure but also has abundant dynamic phenomena, including time-varying stability, bistable, comb-shaped chaotic structures, and coexisting attractors.

The existence condition of the equilibrium point under the external periodic electric field disturbance is given based on the theoretical analysis, and the time-varying stability of the equilibrium point is verified by numerical simulation. Meanwhile, this is also the internal reason for the coexistence of multiple attractors in the model. Importantly, the number of equilibrium points and the stability of the model, as well as saddle-node bifurcation and Hopf bifurcation behaviors, were determined on the key parameter planes (i.e., Figs. 3(a)-(c)) by using the MATCONT package. Interestingly, the model has a bistable structure and hidden firing behaviors near the subcritical Hopf bifurcation, which consists of quiescent state and period-1 and period-2 bursting. Moreover, we focus on the evolution of the bistable structure under the action of the memristive autapse, the results showed that positive (negative) feedback memristive autapse can improve (inhibitory) the stability and anti-interference ability of the system. It is worth mentioning that the counterintuitive dynamic phenomena can be induced by appropriately adjusting the internal parameters of the memristive autapse. In other words, the positive feedback memristive autapse can inhibit the bursting activities of the system, while the negative feedback memristive autapse can promote the firing frequency of the system. Meanwhile, the dynamic mechanism is explained based on fast-slow dynamics analysis. More precisely, the positive feedback memristive autapse reduces the number of spikes per burster by narrowing the distance between the SN point and SH point in the fast subsystem, indicating that the firing frequency decreases. Conversely, the negative feedback memristive autapse increases the number of spikes per burster by enlarging the distance between the SN point and SH point of a burster, which indicates that the firing frequency is increased or the bursting activities are enhanced.

Two-parameter bifurcation analysis is a simple and feasible simulation method to fully understand the global evolution law of the electrical activities of the model. Extensive numerical studies confirm that the system generally has comb-shaped chaotic structures and period-adding bifurcation behavior. Moreover, although the relationship between the firing modes of neurons and their Hamiltonian energy has been widely reported, few studies have been carried out to locate the multi-attractor coexisting regions by analyzing the energy. Accordingly, we prove that it is a reliable method to determine the multistability regions by analyzing the global evolution law of the energy, which also provides an effective way to analyze the initial value sensitivity of complex systems. More importantly, the unidirectional intermittent electrical synaptic coupling controller involving energy is far more efficient than the traditional electrical synaptic coupling controller in the synchronization process of the slave systems with mismatched parameters. This indicates that the energy consumed in the process of realizing the synchronous control of unidirectional coupling systems is reduced and provides a theoretical basis and useful reference value for related engineering applications. Furthermore, we will further design the optimal unidirectional intermittent field (electric or magnetic field) coupling controller involving energy and realize the related control of synchronization of the coupling system and networks with mismatched parameters.

\section{Acknowledgments}

This work is supported by the National Natural Science Foundation of China (Nos. 11962012, 
61863022 and 11961060) and the Key Project of Natural Sciences Foundation of Gansu Province of China (No. 18JR3RA084).

\section{Declarations}

Data availability The data that support the findings of this study are available from the corresponding author upon reasonable request.

Conflict of interest The authors declare that there are no competing interests regarding the publication of this paper.

\section{References}

[1] Lu Q., Yang Z., Duan L., Gu H.G., Ren W.: Dynamics and transitions of firing patterns in deterministic and stochastic neuronal systems. Chaos Solitons Fractals. 40(2): 577-97 (2009)

[2] Ivancevic, T., Jain, L., Pattison, J., Hariz, A.: Nonlinear dynamics and chaos methods in neurodynamics and complex data analysis. Nonlinear Dyn. 56: 23-44 (2009)

[3] Jia, Y., Gu, H.G.: Identifying nonlinear dynamics of brain functional networks of patients with schizophrenia by sample entropy. Nonlinear Dyn. 96(4): 2327-2340 (2019)

[4] Izhikevich, E.M.: Neural excitability, spiking and bursting. Int J Bifurcation Chaos.10(6): 1171-266 (2000)

[5] Izhikevich, E.M.: Dynamical Systems in Neuroscience: The Geometry of Excitability and Bursting. MIT Press, Cambridge, MA (2007)

[6] Yang, M.H., An, S.C., Gu, H.G., Liu, Z.Q., Ren, W.: Understanding of physiological neural firing patterns through dynamical bifurcation machineries. Neuro Report. 17: 995-999 (2006)

[7] Bean, B.: The action potential in mammalian central neurons. Nat Rev Neurosci. 8(6): 451-65 (2007)

[8] Jia, B., Gu, H.G., Xue, L.: A basic bifurcation structure from bursting to spiking of injured nerve fibers in a two-dimensional parameter space. Cogn Neurodyn. 11(2): 1-12 (2017)

[9] Zhao, X., Kim, J.W. Robinson, P.A., Rennie, C.J.: Low dimensional model of bursting neurons. J Comput Neurosci. 36(1): 81-95 (2014)

[10] Gu, H., Pan, B. A four-dimensional neuronal model to describe the complex nonlinear dynamics observed in the firing patterns of a sciatic nerve chronic constriction injury model. Nonlinear Dyn. 81: 2107-2126 (2015)

[11] Ma, J., Tang, J.: A review for dynamics of collective behaviors of network of neurons. Sci. China Technol. Sci. 58(12): 2038-2045 (2015)

[12] Bao, H., Zhu, D., Liu, W., Xu, Q., Chen, M., Bao, B.C.: Memristor synapse-based Morris-Lecar model: mifurcation analyses and FPGA-based validations for periodic and chaotic bursting/spiking firings. Int $\mathbf{J}$ Bifurcation Chaos. 30(3): 2050045 (2020)

[13] Li, K., Bao, H., Li, H.Z., Ma, J., Hua, Z.Y., Bao, B.C.: Memristive Rulkov neuron model with magnetic induction effects. IEEE Transactions on Industrial Informatics. 99: 1-10 (2021)

[14] Wang, Y., Ma, J., Xu, Y., et al.: The Electrical Activity of Neurons Subject to Electromagnetic Induction and Gaussian White Noise[J]. Int J Bifurcation Chaos. 27(2): 1750030 (2017)

[15] Lv, M., Wang, C.N., Ren, G.D., Ma, J., Song, X.L.: Model of electrical activity in a neuron under magnetic flow effect. Nonlinear Dyn. 85: 1479-1490 (2016)

[16] Lv, M., Ma, J.: Multiple modes of electrical activities in a new neuron model under electromagnetic radiation. Neurocomputing. 205: 375-381 (2016)

[17] Wu, F.Q., Wang, C.N., Xu, Y., et al. Model of electrical activity in cardiac tissue under electromagnetic induction. Sci Rep. 6: 28 (2016) 
[18] Bao, H., Hu, A.H., Liu, W.B., et al.: Hidden bursting firings and bifurcation mechanisms in memristive neuron model with threshold electromagnetic induction. IEEE Trans on Neural Netw Learn Syst. 31(2): 502-511 (2019)

[19] An, X.L., Qiao, S.: The hidden, period-adding, mixed-mode oscillations and control in a HR neuron under electromagnetic induction. Chaos Solitons Fractals. 143: 110587 (2021)

[20] Qiao, S., An, X.L.: Dynamic response of the e-HR neuron model under electromagnetic induction. Pramana J Phys. 95: 72 (2021)

[21] Kengne, J., Tabekoueng, Z.N., Tamba, V.K., et al.: Periodicity, chaos, and multiple attractors in a memristorbased Shinriki's circuit. Chaos. 25: 103126 (2015)

[22] Rao, X.B., Zhao, X.P., Gao, J.S., et al.: Self-organizations with fast-slow time scale in a memristor-based Shinriki's circuit. Commun Nonlinear Sci Numer Simul. 94(3): 105569 (2021)

[23] Chen, M., Xu, Q., Lin, Y. et al.: Multistability induced by two symmetric stable node-foci in modified canonical Chua's circuit. Nonlinear Dyn. 87(2): 789-802 (2018)

[24] Zhang, X., Li, C.B., Chen, Y.D., et al.: A memristive chaotic oscillator with controllable amplitude and frequency. Chaos Solitons Fractals. 139: 110000 (2020)

[25] Rao, X.B., Chu, Y.D., Chang, Y.X., et al.: Dynamics of a cracked rotor system with oil-film force in parameter space. Nonlinear Dyn. 88: 2347-2357 (2017)

[26] Liu, Y., Páez Chávez, J.: Controlling multistability in a vibro-impact capsule system. Nonlinear Dyn. 88: 1289-1304 (2017)

[27] Lv, X.H., Zhu, X.F., Gao, Q.F., et al.: Two-parameter bifurcations of an impact system under different damping conditions. Chaos Solitons Fractals. 138: 109972 (2020)

[28] Bao, B.C., Hu, A.H., Xu, Q., et al.: AC-induced coexisting asymmetric bursters in the improved HindmarshRose model. Nonlinear Dyn. 92: 1695-1706 (2018)

[29] Bao, H., Hu, A.H., Liu, W.B.: Bipolar pulse-induced coexisting firing patterns in two-dimensional HindmarshRose neuron model. Int J Bifurc Chaos. 29(1): 1950006 (2019)

[30] Qiao, S., An, X.L.: Dynamic expression of HR neuron model under electric field[J]. Int J Modern Phys B. 35(2): 2150024 (2021)

[31] Rech, P.C.: Multistability in a periodically forced brusselator. Braz J Phys. 51: 144-147 (2021).

[32] Negou, A.N., Kengne, J.: Dynamic analysis of a unique Jerk system with a smoothly adjustable symmetry and nonlinearity: reversals of period doubling, offset boosting and coexisting bifurcations. AEU Int J Electron Commun. 90: 1-19 (2018)

[33] Wang, G., Yuan, F., Chen, G., et al.: Coexisting multiple attractors and riddled basins of a memristive system. Chaos. 28(1):013125 (2018)

[34] Li, C.B., Sprott, J.C.: An infinite 3-D quasiperiodic lattice of chaotic attractors. Phys Lett A. 382(8): 581-587 (2018)

[35] Chen, M., Ren, X., Wu, HG. et al.: Periodically varied initial offset boosting behaviors in a memristive system with cosine memductance. Front Inform Technol Electron Eng. 20(12): 1706-1716 (2019)

[36] Bao, H., Liu, W., Chen, M.: Hidden extreme multistability and dimensionality reduction analysis for an improved non-autonomous memristive FitzHugh-Nagumo circuit. Nonlinear Dyn. 96: 1879-1894 (2019).

[37] Bao, B.C., Qian, H., Xu, Q., et al.: Coexisting Behaviors of Asymmetric Attractors in Hyperbolic-Type Memristor based Hopfield Neural Network. Front Comput Neurosci. 11: 81 (2017)

[38] Bao, B.C., Zhu, Y., Li, C., et al.: Global multistability and analog circuit implementation of an adapting synapse-based neuron model. Nonlinear Dyn. 101: 1105-1118 (2020)

[39] Chen, C.J., Chen, J.Q., Bao, H. et al.: Coexisting multi-stable patterns in memristor synapse-coupled Hopfield 
neural network with two neurons. Nonlinear Dyn. 95: 3385-3399 (2019)

[40] Zhao, Z.G., Li, L., Gu, H.G., et al.: Different dynamics of repetitive neural spiking induced by inhibitory and excitatory autapses near subcritical Hopf bifurcation. Nonlinear Dyn. 99: 1129-1154 (2020)

[41] Ma, H.Q., Jia, B., Li, Y.Y, et al.: Excitability and Threshold Mechanism for Enhanced Neuronal Response Induced by Inhibition Preceding Excitation[J]. Neural Plasticity. 2021(6): 1-18 (2021)

[42] Li, Y.Y., Gu, H., Ding, X.L: Bifurcations of enhanced neuronal bursting activities induced by the negative current mediated by inhibitory autapse. Nonlinear Dyn. 97: 2091-2105 (2019)

[43] Lu, B., Gu, H.G., Wang, X.J., et al.: Paradoxical enhancement of neuronal bursting response to negative feedback of autapse and the nonlinear mechanism. Chaos Solitons Fractals. 145: 110817 (2021)

[44] Wu, F.Q., Gu, H.G., Li, Y.Y.: Inhibitory electromagnetic induction current induces enhancement instead of reduction of neural bursting activities[J]. Commun Nonlinear Sci Numer Simul. 79: 104924 (2019)

[45] Chik, D.T., Wang, Z.D.: Postinhibitory rebound delay and weak synchronization in Hodgkin-Huxley neuronal networks. Phys Rev E. 68(3): 031907 (2003)

[46] Jia Y.B., Gu, H.G., Li, Y.Y., et al.: Inhibitory autapses enhance coherence resonance of a neuronal network. Commun Nonlinear Sci Numer Simul. 95: 105643 (2021)

[47] Wang, X.J., Gu, H.G., Lu, B.: Paradoxical reduction and the bifurcations of neuronal bursting activity modulated by positive self-feedback. Nonlinear Dyn. 101: 2383-2399 (2020)

[48] Wu, F.Q., Gu, H.G.: Bifurcations of negative responses to positive feedback current mediated by memristor in a neuron model with bursting patterns. Int J Bifurcation Chaos. 30(4): 2030009 (2020)

[49] Wu, F.Q., Zhang, Y., Zhang, X.J.: Regulating firing rates in a neural circuit by activating memristive synapse with magnetic coupling. Nonlinear Dyn. 98(2): 971-984 (2019)

[50] Wang, Y., Wang, C.N., Ren, G.D. et al.: Energy dependence on modes of electric activities of neuron driven by multi-channel signals. Nonlinear Dyn. 89: 1967-1987 (2017)

[51] Yang, Y.M., Ma, J., Xu, Y., et al.: Energy dependence on discharge mode of Izhikevich neuron driven by external stimulus under electromagnetic induction. Cogn Neurodyn. 15: 265-277 (2021)

[52] Song, X.L., Jin, W.Y., Ma, J.: Energy dependence on the electric activities of a neuron[J]. Chin Phys B. 24(12): $128710(2015)$

[53] Guo, S.L., Ma, J., Alsaedi, A.: Suppression of chaos via control of energy flow. Pramana J Phys. 90:39 (2018)

[54] A, X.L., Z, L.: Dynamics analysis and Hamilton energy control of a generalized Lorenz system with hidden attractor. Nonlinear Dyn. 94: 2995-3010 (2018)

[55] Wu, F.Q., Hayat, T., An, X.L., et al.: Can Hamilton energy feedback suppress the chameleon chaotic flow? Nonlinear Dyn. 94: 669-677 (2018)

[56] Wang, C.N., Tang, J. Ma, J.: Minireview on signal exchange between nonlinear circuits and neurons via field coupling. Eur. Phys. J. Spec. Top. 228: 1907-1924 (2019)

[57] Hou Z.L., Ma J., Zhan X., et al.: Estimate the electrical activity in a neuron under depolarization field. Chaos Solitons Fractals. 142(4): 110522 (2020)

[58] Ma, J., Zhang, G., Hayat, T., et al.: Model electrical activity of neuron under electric field. Nonlinear Dyn. 95: 1585-1598 (2019)

[59] Yan, B., Panahi, S., He, S. et al. Further dynamical analysis of modified Fitzhugh-Nagumo model under the electric field. Nonlinear Dyn. 101: 521-529 (2020)

[60] Wouapi, K.M., Fotsin, B.H., Louodop, F.P., et al.: Various firing activities and finite-time synchronization of an improved Hindmarsh-Rose neuron model under electric field effect. Cogn Neurodyn. 14: 375-397 (2020)

[61] An, X.L., Qiao, S., Zhang, L.: Dynamic response and control of neuros based on electromagnetic field theory. Acta Phys Sin. 70(5): 46-65 (2021) 
[62] Zhang, G., Guo, D., Wu, F.Q., et al.: Memristive autapse involving magnetic coupling and excitatory autapse enhance firing. Neurocomputing. 379(28): 296-304 (2020)

[63] Dhooge, A., Govaerts, W., Kuznetsov, Y.A.: MATCONT. ACM transactions on mathematical software. 29(2): 141-164 (2003)

[64] Ermentrout, B.: Simulating, analyzing, and animating dynamical systems: a guide to XPPAUT for researchers and students. Appl mech Rev. 56(4): 53 (2003)

[65] Fazanaro, F.I., Soriano, D.C., Suyama, R., et al.: Numerical characterization of nonlinear dynamical systems using parallel computing: The role of GPUs approach. Commun Nonlinear Sci Numer Simul. 37: 143-162 (2016)

[66] Xu, Y., Ying, H.P., Jia, Y., et al.: Autaptic regulation of electrical activities in neuron under electromagnetic induction[J]. Sci Rep. 7: 43452 (2017)

[67] Wang, C.N., Wang, Y., Ma J.: Calculation of Hamiltonian energy function of dynamic system by using Helmholtz theorem. Acta Phys Sin. 65(24): 240501 (2016)

[68] Zhou, P., Hu, X.K., Zhu, Z.G., et al.: What is the most suitable Lyapunov function? Chaos Solitons Fractals. 150: 111154 (2021) 